\title{
New Approach to Evaluate the Lubrication Process in Various Granule Filling Levels and Rotating Mixer Sizes Using a Thermal Effusivity Sensor
}

\author{
Jumpei Uchiyama, ${ }^{* a}$ Shigeru Aoki, ${ }^{a}$ and Yoshifumi Uemoto ${ }^{b}$ \\ ${ }^{a}$ Japan Technology, Global Demand Chain Technology, New Chemical Entity Demand Chain Unit, Eisai Demand \\ Chain Systems, Eisai Co., Ltd.; 1 Kawashimatakehaya, Kakamigahara, Gifu 501-6195, Japan: and ${ }^{b}$ Global \\ Formulation Japan Pharmaceutical Science Technology, Eisai Co., Ltd.; 1 Kawashimatakehaya, Kakamigahara, Gifu \\ 501-6195, Japan. \\ Received September 4, 2014; accepted January 5, 2015
}

The principles of thermal effusivity are applied to an understanding of the detailed mechanisms of the lubrication process in a rotating mixer. The relationships and impact of the lubrication process by the pattern of powder flow, the filling level, and the rotating mixer size were investigated. Thermal effusivity profiles of the lubrication process, as obtained, indicate that lubrication is a two-phase process. The intersection point of the first and second phases (IPFS) is influenced by changing the filling level, thus changing the resulting number of avalanche flows created. The slope of the second phase (SSP) is influenced by the relationship between the number and the length of avalanche flows. Understanding this difference between the first and second phases is important to successfully evaluate the impact of proposed changes in the lubrication process. From this knowledge, a predictive model of the lubrication profile can be generated to allow an evaluation of proposed changes to the lubrication process. This model allows estimation of the lubrication profile at different filling levels and in different rotating mixer sizes. In this study, the actual lubrication profile almost coincides with the model predicted lubrication profile. Based on these findings, it is assumed that lubrication profiles at a commercial scale can be predicted from data generated at the laboratory scale. Further, it is assumed that changes in the filling level can also be estimated from the laboratory or current data.

Key words lubrication process mechanism; process analytical technology; thermal effusivity; monitoring lubrication process; magnesium stearate

Compressed tablets are a very popular and important pharmaceutical dosage form. The manufacturing of tablets is a complex processes, because the physical properties of the granules used in compression are critical to success and these properties can be changed by various factors. The size distribution of the granules, the formulation and the materials used in manufacturing tablets can have a direct impact on the quality of the tablets. A well thought out development plan is needed to achieve a final formulation and robust manufacturing process to manufacture tablets that meet specifications.

Wet granulation is the most common process used to make the granules for tablet manufacturing. The process flow of wet granulation consists of the following steps before compression ${ }^{1)}$ : mixing (active ingredient(s) and diluents), wetting (binder and water), granulation, drying, sieving and blending (glidant, lubricant and disintegrant). Each process step is monitored and controlled to assure the quality of the tablets produced. Various monitoring techniques have historically been used at each process step. These techniques are very helpful during any investigations or for resolving difficulties during drug development or observed during scale-up from the laboratory scale to the commercial scale. A review of current literature provides a description of the granulation process, ${ }^{2)}$ as well as various reports related to monitoring ${ }^{3,4)}$ the granulation process and investigations related to scale$u^{5-8)}$ that have been published. The lubrication process is also discussed in the literature and has been studied by various approaches. The lubrication process involves the blending of a minor constituent and is often seen as a very simple op- eration. The desired outcome of the lubrication process is to disperse the lubricant and to provide adequate coating of the powder particles with the lubricant..$^{9-11)}$ In the formulation, the selected lubricant is one of the most important excipients. The lubricant is responsible for the lubricating (lubricity) the granules and allowing them to flow easily. The lubricant can often be the source of difficulties during the tableting process and can have negative impacts on tablet properties. ${ }^{9,10,12-14)}$ An adequate combination of the lubricant concentration and then blending time is important to assure tablet quality attributes are achieved, and that the tableting process is robust and capable of high-speed manufacturing. Magnesium stearate ${ }^{15-17)}$ is one of the more common lubricants used in tablet manufacturing and is often used in very low concentrations $(0.2-2.0 \%)$. Optimization studies designed to evaluate the lubricant concentration and the blending conditions are often required to achieve the target tablet quality. If the lubricant concentration is too low or if the blending time is inadequate, the powder will often stick to the tablet press punches, or the tablets may bind in the die cavity. These events result in excessive ejection forces during tableting that often leads to breakage of tablets. The tablets might also show signs of picking or sticking as a result of the adhesion to the punch faces. Having a lubricant concentration that is too high or a blending time that is too long can lead to other problems, such as excessive coating of the powder with the lubricant. This can lead to insufficient tablet hardness, extension of tablet disintegration times and delays in dissolution rates. ${ }^{18,19)}$ Therefore, the control of the blending with lubricant is critical to various aspects of the 
process and to the final tablet properties.

There are many kinds of powder mixers ${ }^{20)}$ used to conduct the blending process. The blending mechanism has been studied previously using various approaches, ${ }^{21-26)}$ but these studies mainly evaluated normal blending that included the blending of only two components (typically in equal proportion) and focused on the blend homogeneity. The lubrication process is different, because we are attempting to blend a minor constituent, to disperse the lubricant and to provide coating of the powder particles. The detailed mechanism of lubricant blending has not been fully studied and is not as well understood. There have been several reports published that describe methods for the scale-up of the lubrication process using various approaches. ${ }^{27-32)}$ These former studies were mainly focused on an evaluation of the tablet properties and were not focused on lubrication process itself.

More recently, blending and other processes are being monitored using new strategies such as process analytical technology (PAT) coupled with tools like near infrared (NIR) spectroscopy, thermal effusivity and others. ${ }^{33)}$ PAT has been investigated for use in producing pharmaceutical granules during continuous processing. ${ }^{34-36)}$ NIR is most often the chosen method to analyze the homogeneous blending process, ${ }^{37-42)}$ the lubrication process, ${ }^{42-47)}$ the tableting process ${ }^{48-50)}$ and the film coating process. ${ }^{51-53)}$ Thermal effusivity ${ }^{54-60)}$ is another technique that has potential PAT applications and is capable of monitoring the lubrication process. Terashita ${ }^{59)}$ conducted a comparative investigation of the lubrication process using both NIR and thermal effusivity. The Terashita's study concluded that the end points of the lubrication process using NIR and the thermal effusivity were different. Terashita reported that the end point of the lubrication process using the NIR method (1 min) was earlier than when using the thermal effusivity method (between 2 and $3 \mathrm{~min}$ ). The different results with each method could be attributed to different phenomena (dispersion and coating by lubricant). The NIR result was proposed to be mainly indicative of the dispersion of the lubricant, and the thermal effusivity results were proposed to be indicative of the dispersion of the lubricant and coating of the powder with the lubricant. A thermal effusivity sensor can detect the physical change in the bulk density caused by the dispersion of lubricant and the coating of the powder particles with lubricant. ${ }^{61)}$ The ability of the thermal effusivity to detect both phenomena makes this an interesting option for investigation of the lubrication process. The thermal effusivity sensor has the potential to reveal the detailed mechanism of the lubrication process in a new and different way.

There are our two reports ${ }^{61,62)}$ regarding the detailed investigation of the lubrication process using the thermal effusivity sensor. Firstly, our previous report ${ }^{61)}$ indicates that the thermal effusivity is a useful tool for PAT monitoring to estimate tablet hardness and for investigating the detailed mechanism of lubrication process. Since the monitoring of thermal effusivity refers that of bulk density, the thermal effusivity sensor was able to have a high correlation with tablet hardness. Moreover, the two-phase progress of the lubrication process was revealed in this study because the thermal effusivity sensor could detect not only a fractional change of the conventional bulk density but also a fractional change of thermal conductivity and thermal capacity. The monitoring of more detailed lubrication process was possible by using thermal effusivity sensor. Sec- ondary, for investigating the detailed mechanism of lubrication process when the blending condition of the lubrication process was changed in the various rotation speeds and the filling levels, our previous report ${ }^{62)}$ indicates that the number of revolutions was more important than the rotation speed, and the critical rotation speed (CRS) is determined based on the filling level. In the monitoring by the thermal effusivity sensor in the region of the lower CRS, the progress of lubrication per the number of lubrication process was the same, regardless of the rotation speed. In addition, the detailed progress of the lubrication progress was shown to be a two-phase process based on the analysis of the profile of the thermal effusivity. The first phase was influenced by the CRS and the filling level in the blender. The second phase was influenced by the rotation speed. The mechanism of two-phase process was proposed as a macro progression of the dispersion of the lubricant (first phase) and micro progression of the coating of the powder particles with lubricant (second phase). When the blending condition of the lubrication process was changed, it is necessary to pay attention to the influence of each phase.

In this study, by adding newly the investigation of influence on filling level and the rotating mixer size in lubrication process to our previous knowledge, ${ }^{61,62)}$ the detailed mechanism of lubrication process should be discussed in a comprehensive way. This is because the previous reports ${ }^{61,62)}$ did not sufficiently discuss the rotating mixer size in the lubrication process and did not sufficiently investigate the factors that the change of filling level has impact on the difference of lubrication process. Better understanding of the effects that the filling level and the rotating mixer size have on the process may help clarify the detailed mechanism of the lubrication process more than ever. The knowledge gained could help resolve problems often seen with changes of the lubrication conditions or in the rotating mixer size in pharmaceutical manufacturing. Especially, taking into account the rotating mixer size is very important for scale-up study from the laboratory scale to the commercial scale. Moreover, using knowledge of these, we tried to establish a model of the lubrication process which could be applied to predict the lubrication profile in consideration of the important and controllable various parameters (rotation speed, filling level and rotating mixer size).

\section{Experimental}

Materials Lactose (Tablettose 80, Meggle G.m.b.H., Germany) and Magnesium stearate (Mallinckrodt Japan Co., Ltd., Japan) were used as filler and lubricant.

Operation of the Lubrication Blending Lactose (99.8\%) and Magnesium stearate $(0.2 \%)$ were blended using horizontal drum type tumble mixers (Showa Kagakukikai Kosakusho Co., Ltd., Japan) of various sizes. The mixers and models included a TM-20 mixer (volume; 20L, diameter; $0.370 \mathrm{~m}$ ), a TM-50 mixer (volume; 50 L, diameter; $0.480 \mathrm{~m}$ ) and a TM-100 mixer (volume; $100 \mathrm{~L}$, diameter; $0.628 \mathrm{~m}$ ). The rotation speed for each mixer was set at slightly different values so that the Froude Number was the same. To achieve this, the speeds were $18.7 \mathrm{rpm}$ (TM-20 mixer), $16.4 \mathrm{rpm}$ (TM-50 mixer) and $14.3 \mathrm{rpm}$ (TM-100 mixer). The filling levels evaluated are described in terms of the percentage of the maximum amount of blend for each mixer size and also the absolute amount $(\mathrm{kg})$. The filling levels evaluated in the TM-20 mixer were 8.8, 17.5, 35.1, 52.6, 70.2 and $87.7 \%$ using blend amounts of 1.0, 2.0, 4.0, 
(a)

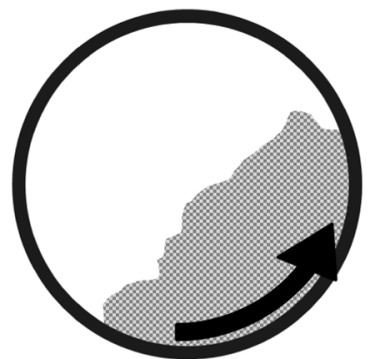

Uplifted of powder

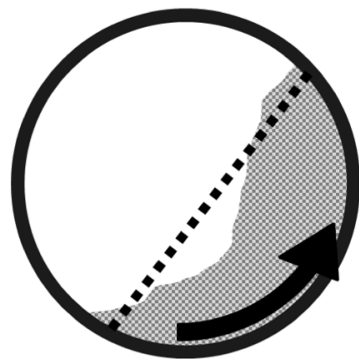

Before avalanche flow

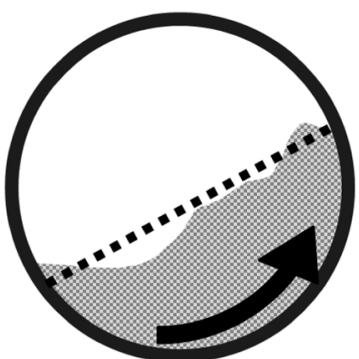

After avalanche flow

(b)

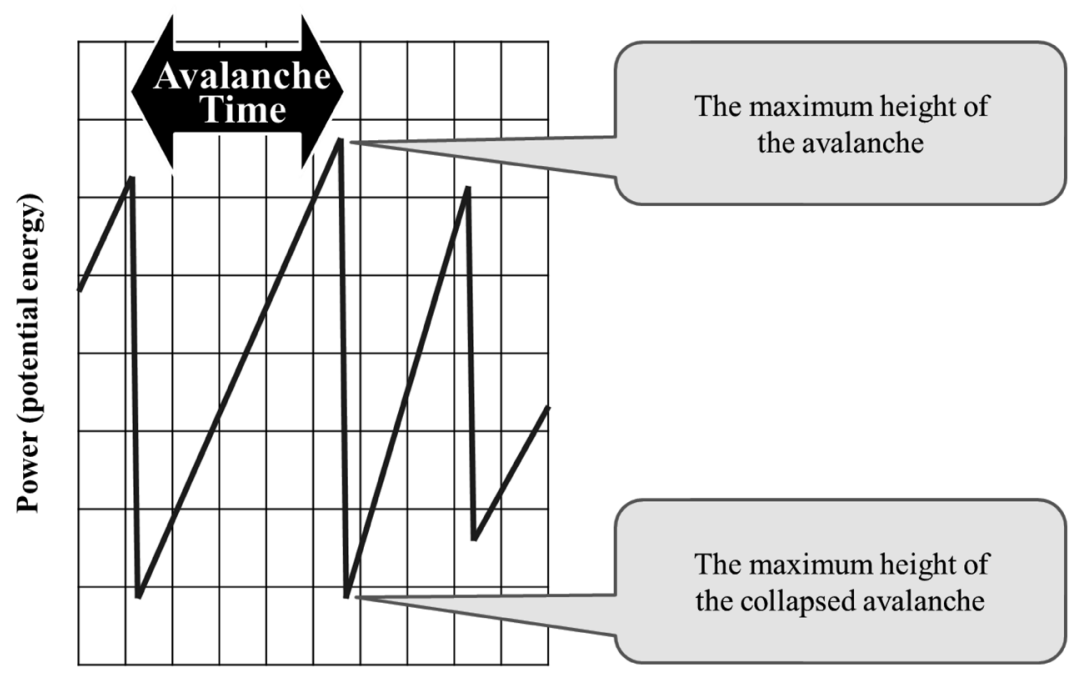

Elapsed time [sec]

Fig. 1. Image of Measurement of Avalanche Time

$6.0,8.0$ and $10.0 \mathrm{~kg}$, respectively. The filling levels evaluated in the TM-50 mixer were 8.8, 35.1, 52.6 and $87.7 \%$ using blend amounts of $2.5,10.0,15.0$ and $25.0 \mathrm{~kg}$, respectively. The filling levels evaluated in the TM-100 mixer were 8.8, 35.1, 52.6 and $87.7 \%$ using blend amounts of $5.0,20.0,30.0$ and $50.0 \mathrm{~kg}$, respectively.

The blender was stopped at predefined intervals so that a $10 \mathrm{~g}$ sample of the blended powder could be taken and analyzed using the thermal effusivity sensor. This sample was taken from the center of the blender at a location approximately $1 \mathrm{~cm}$ underneath the top of the powder layer using a $20 \mathrm{~mL}$ cup. The blending process was stopped when no further changes in thermal effusivity were observed.

Measurement of the Thermal Effusivity The thermal effusivity is calculated from Eq. 1;

$$
E=\sqrt{k \rho C_{p}}
$$

where $E, k, \rho$ and $C_{p}$ are thermal effusivity $\left(\mathrm{Ws}^{1 / 2} / \mathrm{m}^{2} \mathrm{~K}\right)$, thermal conductivity $(\mathrm{W} / \mathrm{m} \cdot \mathrm{K})$, bulk density $\left(\mathrm{kg} / \mathrm{m}^{3}\right)$ and heat capacity $(\mathrm{J} / \mathrm{kg} \cdot \mathrm{K})$, respectively.

A Thermal Effusivity Sensor (Mathis ESP-04, C-therm Technologies, Canada) was used to measure the thermal effu- sivity. A ten gram sample of the powder taken from the mixer was measured (off-line). The analyses were performed using an interval time of $60 \mathrm{~s}$ and a measuring time of $1.5 \mathrm{~s}$. The thermal effusivity result reported is expressed as the average of the three measurements.

Measurement of Avalanche Time A REVOLUTION ${ }^{\circledR}$ Powder Analyzer (Mercury Scientific Inc., U.S.A.) was used to evaluate the avalanche properties of the powder. The important avalanche flow properties of a powder include the avalanche time, the avalanche angle, the avalanche power, the rest angle, the surface Linearity and others. ${ }^{63,64)}$ The powder to be tested was placed inside the rotating drum of the RPA $\left(\right.$ REVOLUTION $^{\circledR}$ Powder Analyzer) and measurements were made at various filling levels. The instrument diameter of the rotating drum was $0.100 \mathrm{~m}$ and the width is $0.035 \mathrm{~m}$.

At each filling level, the RPA was run until 128 avalanches had occurred. Figure 1a shows cartoons of an avalanche cycle showing the powder in the drum at different stages leading to and following an avalanche. Figure $1 \mathrm{~b}$ shows the avalanche cycle on a time scale and how the avalanche time can be calculated as the duration of each avalanche cycle (time from start to end of each avalanche flow). The average avalanche 


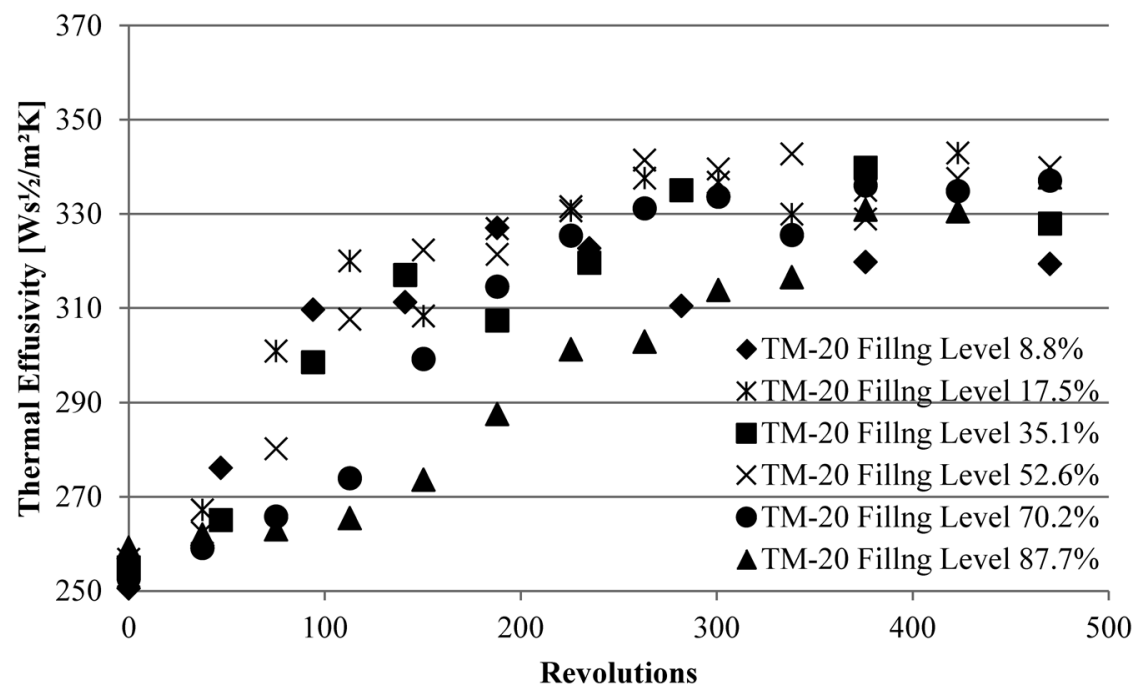

Fig. 2. Thermal Effusivity Data throughout the Lubrication Process

TM-20 mixer; the filling level=8.8, 17.5, 35.1, 52.6, 70.2 and 87.7\%.

time can be calculates as the sum of each avalanche time divided by total number of avalanche flow and is shown in Eq. 2.

$$
\begin{aligned}
& \text { Avalanche time } \\
& =\frac{\text { sum of the time from start to end in each avalanche }}{\text { total number of avalanche }}
\end{aligned}
$$

The RPA test were conducted at filling levels equivalent to 10 , $20,30,40,50,60,70,80$ and $90 \%$ using actual blend amounts of $16,31,47,63,78,94,110,125$ and $141 \mathrm{~g}$, respectively. The drum speed for each run was set at the standard rotation speed (0.6 rpm) for each RPA test. To understand the powder flow in the blender more easily, lactose alone was used for the RPA test. Magnesium stearate (lubricant) was not added to the RPA, because the addition of a lubricant would be expected to have an impact on the avalanche flow over time.

Analysis of the Profile Obtained by the Thermal Effusivity Sensor The profile generated by the thermal effusivity sensor is a sigmoidal curve and can be analyzed in detail using various methods. Generally, these profiles of sigmoidal type are called growth curves and there are three kinds of curves. They are referred to as the cumulative normal distribution curve (probit transformation), the logistic curve (logit transformation) and the Gompertz curve (difference of translation to logarithm). The cumulative normal distribution curve and logistic curve data analysis methods are typically applied to datasets where the curve is symmetrical about the inflection point. The Gompertz curve data analysis method is typically applied to datasets where the curve is not symmetrical about the inflection point. The Gompertz curve is often applied in many cases to fit the growth curve which increases slowly at first, then increases rapidly from the middle and approaches a limit value. In this study, the Gompertz curve methodology was applied to the sigmoidal function monitored by the thermal effusivity sensor. The thermal effusivity profile was suitable for the application of the Gompertz curve methodology.

The Gompertz curve data analysis method was applied to the sigmoidal function monitored by the thermal effusivity sensor as described in the previous report. ${ }^{62)}$ A difference between the thermal effusivity value and the maximum values was plotted as a semilogarithmic graph as a function of the number of revolutions. Using this method helps to understand the two-phase progress in the lubrication process. The detailed analysis of the thermal effusivity data was able to reveal the detailed mechanism of the lubrication process as a two-phase process.

\section{Results and Discussion}

The Change in the Lubrication Process at Various Filling Levels and Rotating Mixer Sizes The change in thermal effusivity during the lubrication process in the TM-20 mixer at various filling levels is shown in Fig. 2. The initial thermal effusivity was approximately $255 \mathrm{Ws} / 1 / 2 \mathrm{~m}^{2} \mathrm{~K}$ and reached a final constant value of approximately $335 \mathrm{Ws}^{1 / 2} / \mathrm{m}^{2} \mathrm{~K}$. This result was almost the same as reported in other studies. ${ }^{58,59)}$ Roy and Mathis ${ }^{58)}$ reported that the thermal effusivity rose from 270 to $340 \mathrm{Ws}^{1 / 2} / \mathrm{m}^{2} \mathrm{~K}$ with blending time, when a mixture of lactose, microcrystalline cellulose and magnesium stearate $(2.0 \%)$ was blended in a $325-\mathrm{L}$ bin container. Terashita ${ }^{59)}$ reported that the thermal effusivity rose from 270 to $290 \mathrm{Ws}^{1 / 2} / \mathrm{m}^{2} \mathrm{~K}$ during the lubrication process, when a mixture of acetaminophen, lactose and magnesium stearate $(1.0 \%)$ was blended in a high-speed mixer. In current study (shown in Fig. 2), as the filling levels increased, these profiles of the thermal effusivity versus number of revolutions in lubrication process progressed gradually. The profile at $87.7 \%$ filling level progressed more gradually than the other filling levels. The thermal effusivity end point value at the $8.8 \%$ filling level was lower than at the other filling levels. Adherence of the lubricant to the interior wall was observed when the filling level was too low. The powder flow of this case was not considered because the filling level was too low to be compared to other filling levels. The amount of lubricant was slightly decreased in the lubrication process by the influence of this issue. It is speculated that adhesion on the walls resulted in the lower endpoint value for the $8.8 \%$ filling level compared to the other filling levels.

In a similar way, we investigated the changes of the thermal effusivity in the other rotating mixer sizes (TM-50 and TM-100 mixer). The changes of the thermal effusivity profiles with the increasing sizes are shown in Figs. 3 and 4. Similar 


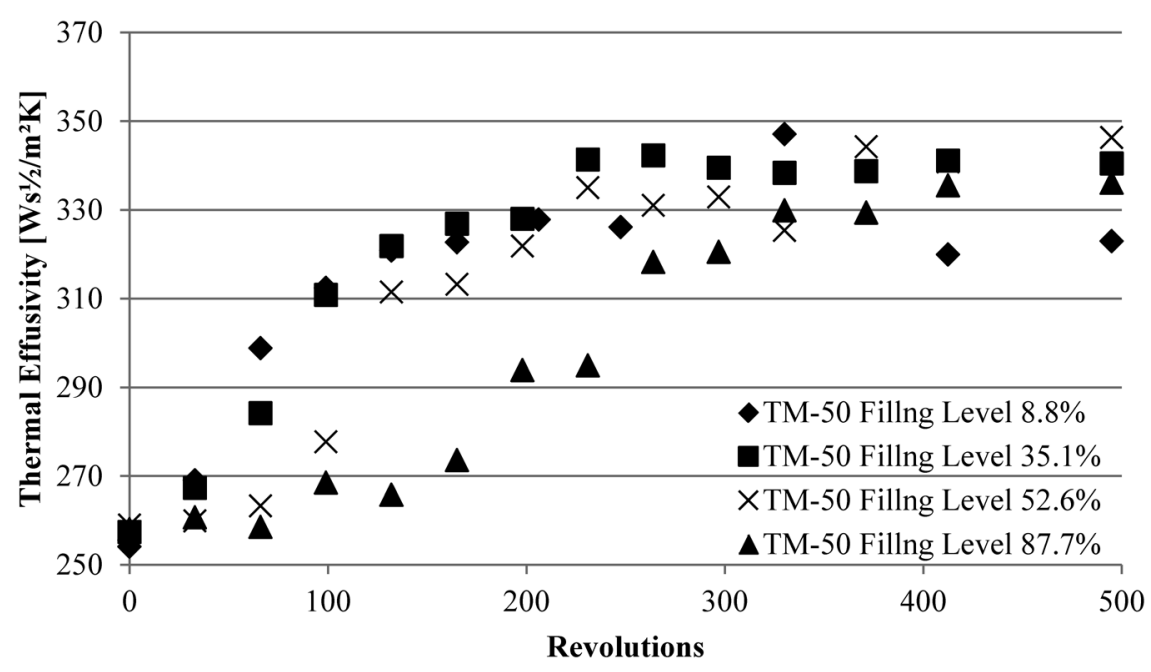

Fig. 3. Thermal Effusivity Data throughout the Lubrication Process

TM-50 mixer; the filling level=8.8, 35.1, 52.6 and $87.7 \%$.

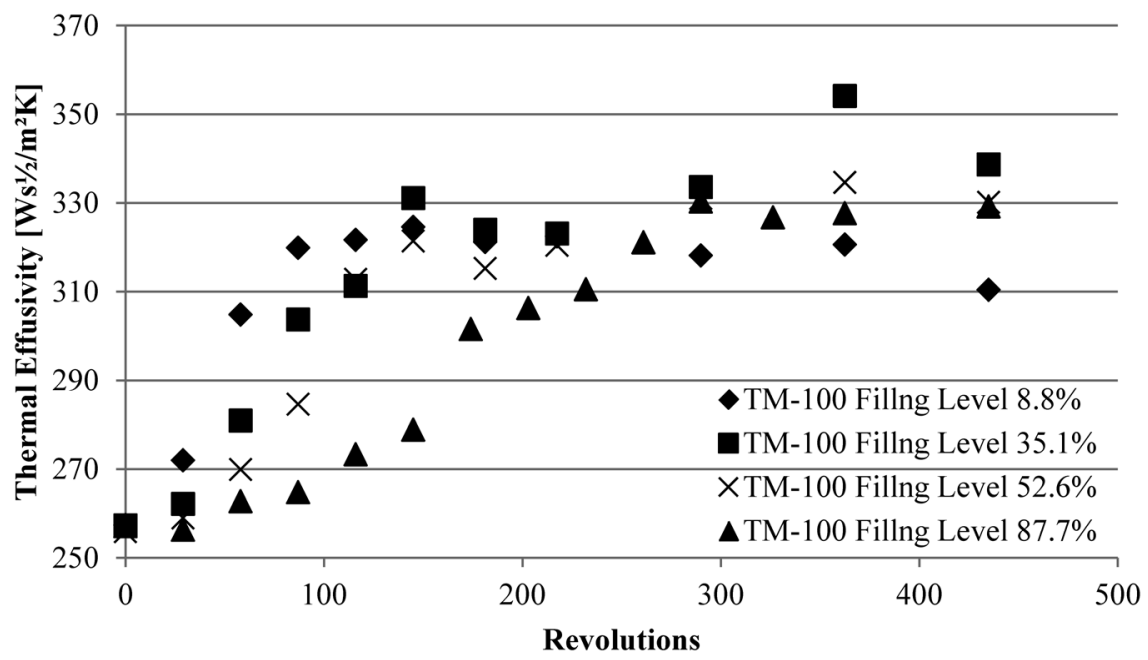

Fig. 4. Thermal Effusivity Data throughout the Lubrication Process

TM-100 mixer; the filling level=8.8, 35.1, 52.6 and $87.7 \%$.

to the results in the TM-20 mixer, the initial thermal effusivity was approximately $255 \mathrm{Ws}^{1 / 2} / \mathrm{m}^{2} \mathrm{~K}$ and reached to a final constant value of approximately $335 \mathrm{Ws}^{1 / 2} / \mathrm{m}^{2} \mathrm{~K}$. This thermal effusivity profiles are almost the same as that of TM-20 mixer, regardless of the rotating mixer sizes. These profiles of the lubrication process progressed gradually as the filling levels increased.

Changes in the thermal effusivity at different filling levels were investigated at the different rotating mixer sizes (TM-20, TM-50 and TM-100 mixer). The profiles in each filling level, 8.8, 35.1, 52.6 and 87.7\% are shown in Figs. 5, 6, 7 and 8, respectively. The profiles of thermal effusivity as a function of revolution in these rotating mixers were almost coincided with each other. At each filling level, the number of revolutions required to obtain the same outcome in the lubrication process were very close, regardless of the rotating mixer sizes.

In our previous report, ${ }^{61,62)}$ the lubrication process was described as a two-phase process, the first phase is the process of the lubricant dispersion. The thermal effusivity sensor can mainly detect the change of the bulk density. ${ }^{61)}$ In addition, the thermal effusivity sensor can also detect fractional changes of thermal conductivity and the thermal capacity, but these changes have comparatively small effect on the thermal effusivity sensor. As the dispersion of the lubricant progresses in the first phase, the thermal conductivity and thermal capacity in the mixture are changed but the bulk density is not changed significantly. Therefore, the value of the thermal effusivity in this first phase is nearly-unchanged. ${ }^{61)}$ For the efficient dispersion of the lubricant, adequate powder flow (avalanche flow) in the first phase is desirable in the rotating mixer. The main purpose of second phase is to coat the powder particles with the lubricant. The value of thermal effusivity during the second phase is changed and increased compared to the first phase. As the powder particles become coated by the uniformly dispersed lubricant, the bulk density increases and the value of the thermal effusivity increases. Powder movement in the rotating mixer is sufficient to coat the powder particles with lubricant in the second phase.

In the current study, the lubrication process was affected by the filling levels but not the rotating mixer size. The profiles 


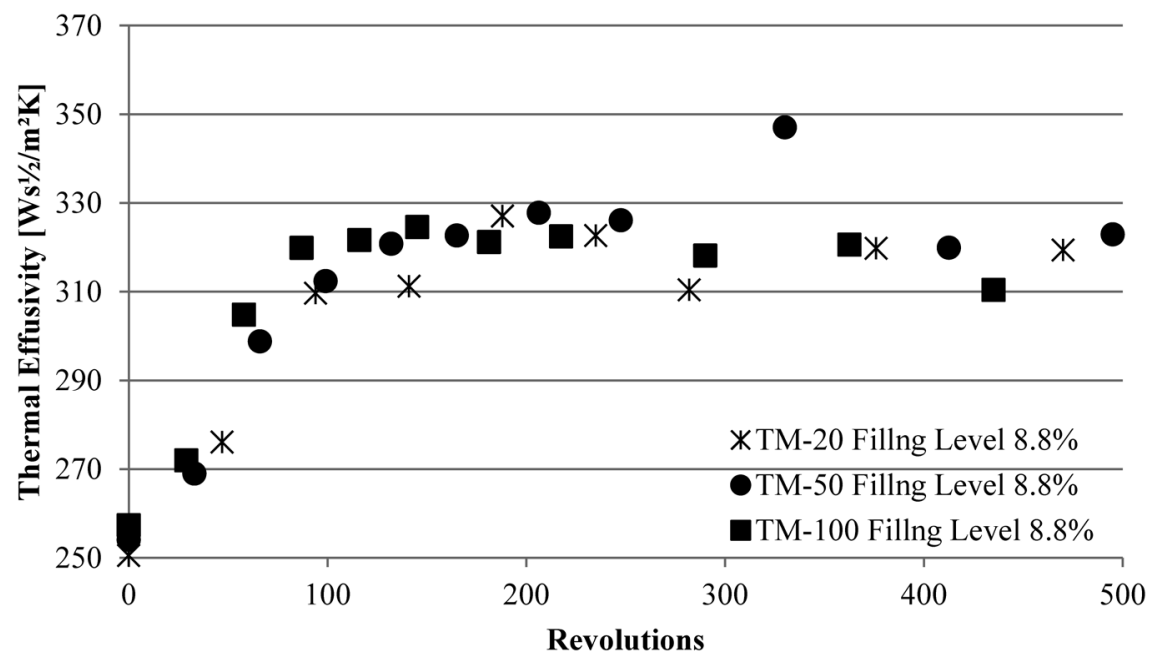

Fig. 5. Thermal Effusivity Data throughout the Lubrication Process

TM20, TM-50 and TM-100 mixer; the filling level $=8.8 \%$.

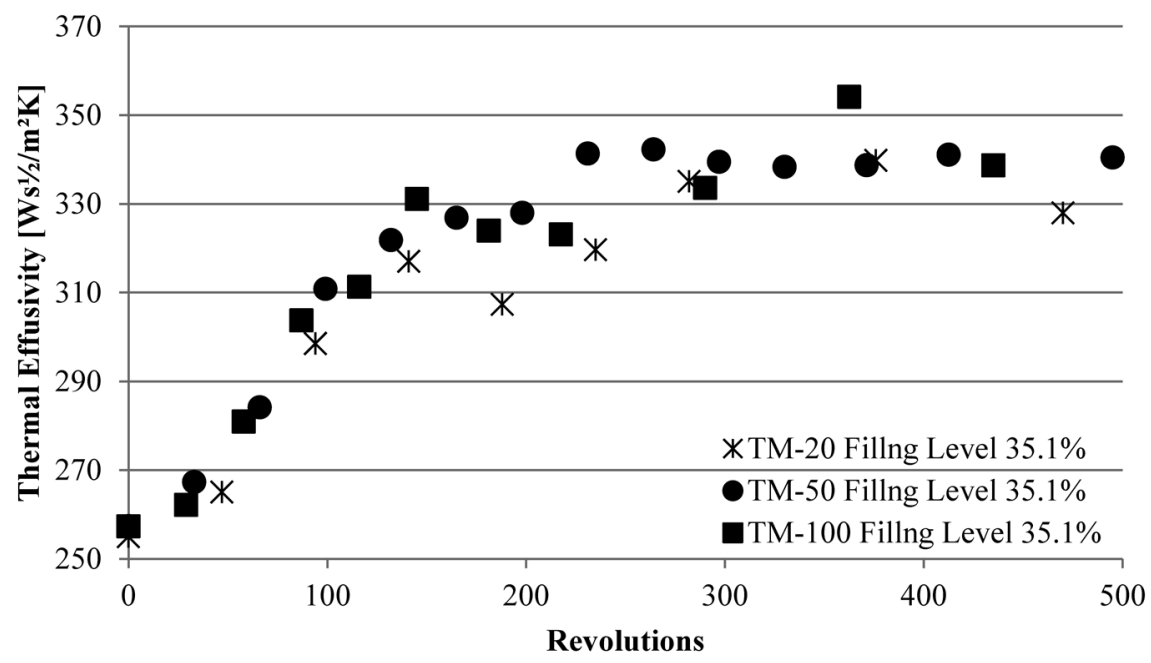

Fig. 6. Thermal Effusivity Data throughout the Lubrication Process

TM20, TM-50 and TM-100 mixer; the filling level $=35.1 \%$.

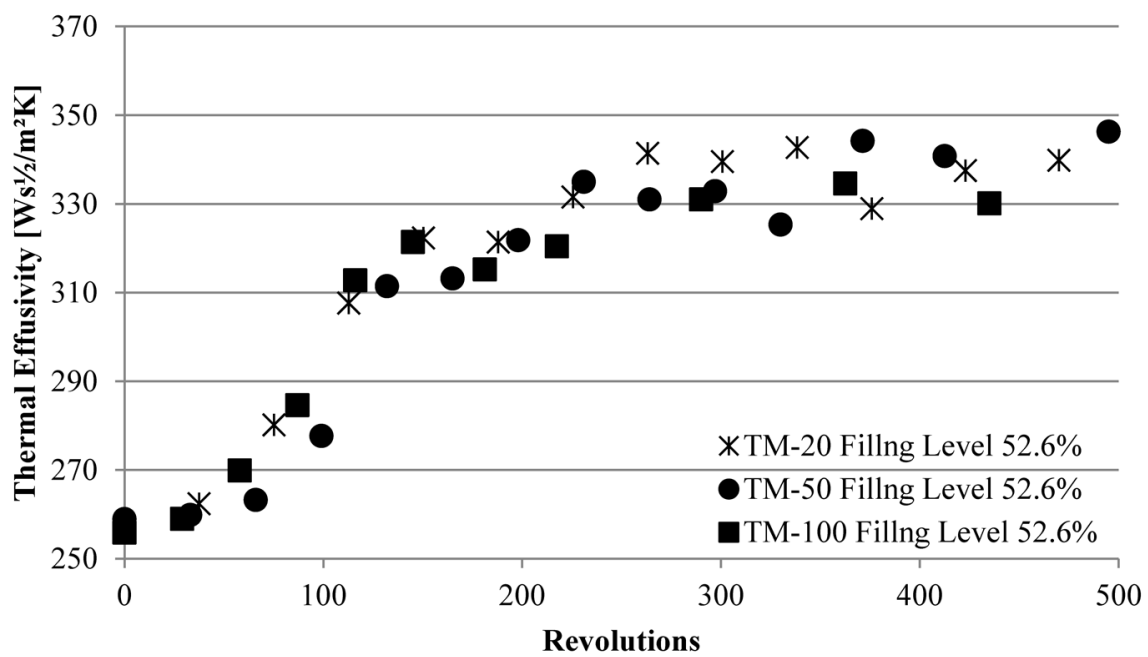

Fig. 7. Thermal Effusivity Data throughout the Lubrication Process

TM20, TM-50 and TM-100 mixer; the filling level $=52.6 \%$. 


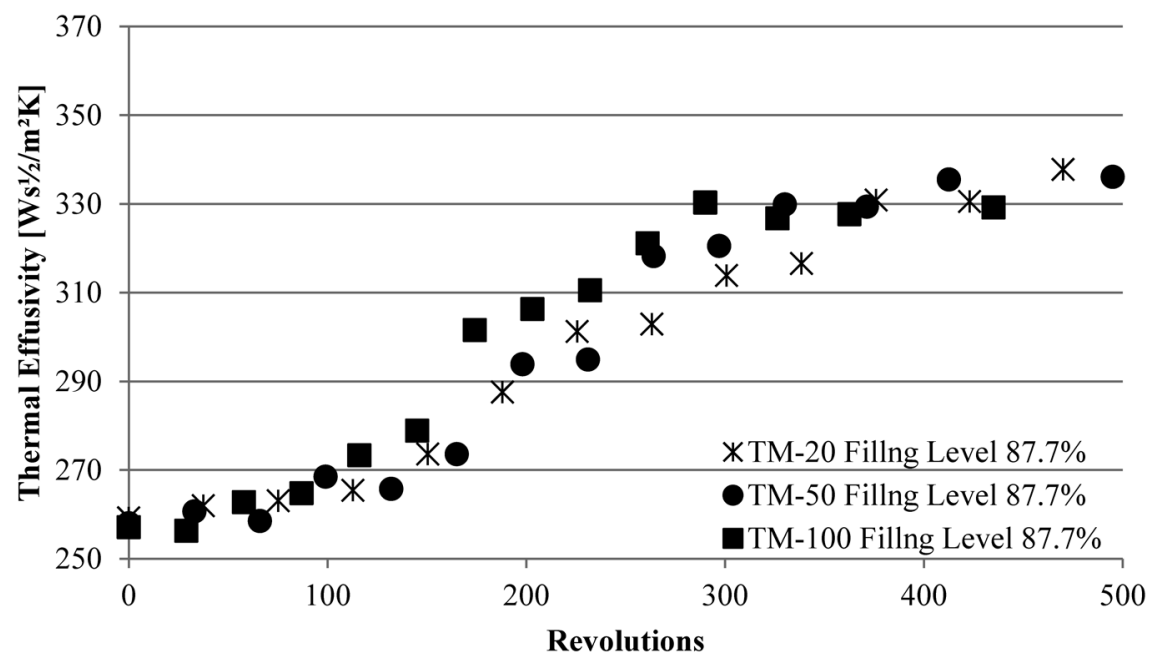

Fig. 8. Thermal Effusivity Data throughout the Lubrication Process

TM20, TM-50 and TM-100 mixer; the filling level $=87.7 \%$.

of the thermal effusivity at various filling levels and various rotating mixer sizes were analyzed in terms of the two-phase process. The relationship between the lubrication process, the filling level and the rotating mixer size should be able to be analyzed by the profile monitored with the thermal effusivity sensor. We investigated the lubrication process by analyzing of the lubrication profile monitored with the thermal effusivity sensor and also the powder flow in the rotating mixer during the lubrication process.

Relationship between the Lubrication Process and the Powder Flow When monitoring the lubrication process by the thermal effusivity sensor, it was noted that these profiles were different in various filling levels. To understand this better, the powder flow in the rotating mixer during the lubrication process was investigated in detail.

The RPA is able to analyze dynamic powder flow properties under stress conditions. Analysis was performed using an image obtained from a digital camera able to take photos of the powder flow during the rotation process. The RPA measures the powder's ability to flow, consolidate, granulate, cake, pack and fluidize by measuring the power, time and variances in energy of the powder in a rotating drum. These data can be used to quantify the powder's particle behavior during process applications such as blending, tableting, mixing and transportation. The average avalanche time, repose angle and the avalanche power were calculated using data generated by the RPA. In general, when the avalanche time, the repose angle and the avalanche power decreased, the powder has high flow-ability. In previous reports, ${ }^{62}$ the relationships between the avalanche flow (avalanche power), the rotation speed and filling level were investigated. The avalanche flow is required for the adequate lubrication process. It was reported that the powder flow in the rotating mixer was avalanche flow. To research powder flow properties, the filling levels of the RPA were changed to investigate the changes in the photo image and the influence on avalanche time.

By using avalanche time obtained from the photos taken of the RPA, the influence of the filling levels (10, 20, 30, 40, $50,60,70,80$ and 90\%) was investigated in detail. To understand the powder flow in the rotating mixer more easily, lactose alone was used for the RPA test as not to demonstrate lubrication effect during the measurement. The result of measurement in avalanche time is shown in Fig. 9. As the filling level increased, the frequency of avalanche flow decreased and avalanche time increased (change 2.1 to $4.3 \mathrm{~s}$ ). The data shows avalanche time was linearly-correlated positively with the filling level.

Inoue's report ${ }^{65)}$ describes a mixing zone and a stagnant zone in a horizontal drum mixer (Fig. 10). The lubrication process in the mixing zone occurs by slipping of the superficial layer. Mixing does not occur in the stagnant zone when the powder is rotated together with the wall of the rotating mixer. For an adequate lubrication process, the avalanche flow which involves slipping on superficial layer is important. The proportion of the mixing zone and the stagnant zone are determined by the filling levels and this has a significant impact on mixing efficiency. To research the mechanism in lubrication process, the profile obtained by thermal effusivity were analyzed in detail by consideration of the mixing zone, frequency of avalanche flow and the two-phase process.

Analysis of the Lubrication Process in Terms of TwoPhase Process The profiles of the lubrication process monitored by the thermal effusivity sensor have been shown to be a tool to analyze the process in detail from the point of a twophase process. The Gompertz curve data analysis method was applied to the sigmoidal function monitored by the thermal effusivity sensor as described in the previous report. ${ }^{62)}$ This method allows us to understand the two-phase process in the lubrication process.

Figure 11 shows the result of analysis of the profiles from the TM-20 mixer. The values in the first phase slightly decrease until the point where the process transitions into the second phase. After the end of the first phase, the thermal effusivity values decrease significantly in the second phase. The profiles at the $8.8 \%$ and $17.5 \%$ filling level were not included in this figure, because the lubrication process was observed to be a one-phase process (only the second phase). It was speculated that high mixing efficiency and the fast progression occurred, because the absolute amount of the powder was small and the occupied volume of powder in the rotating mixer was 


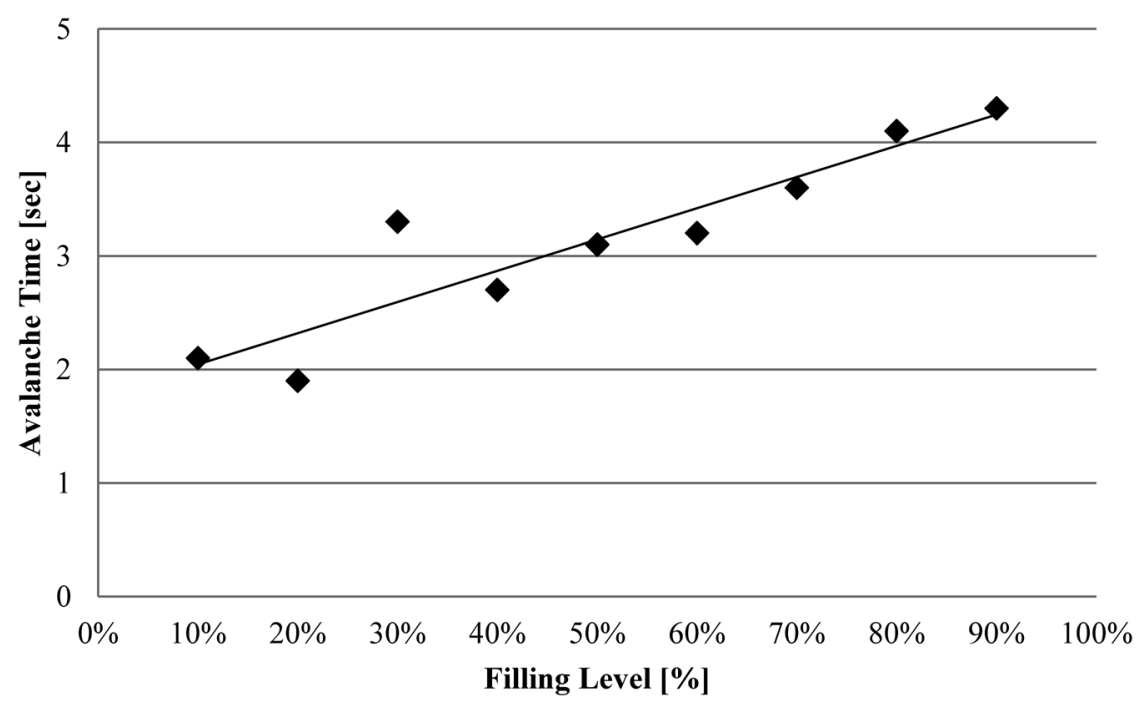

Fig. 9. Avalanche Time by the RPA at the Various Filling Level

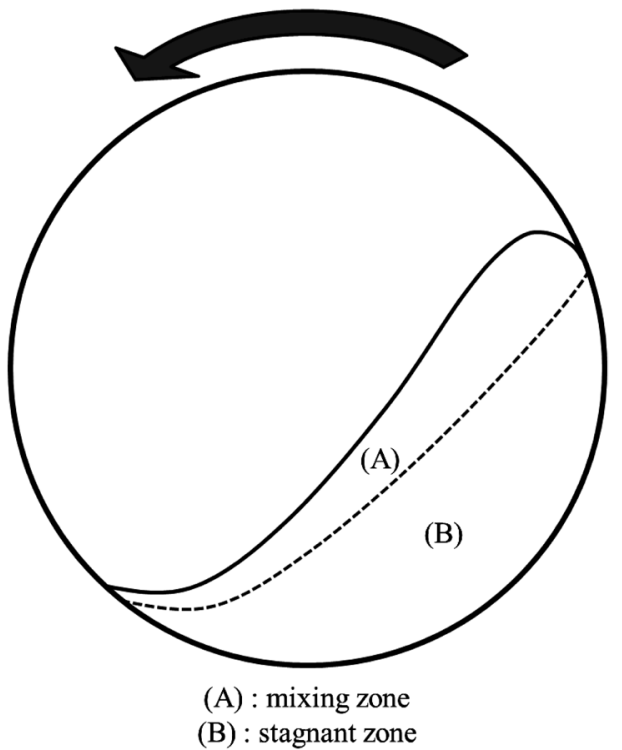

Fig. 10. Image of Mixing Zone and Stagnant Zone in the Blender

very low. Therefore, the second phase alone was observed without the first phase. As filling level increased, the intersection point of the first and the second phases (IPFS) required a larger number of revolutions. The results from the TM-50 and TM-100 mixer were similar to those from the TM-20 mixer. Similar to the results in the TM-20 mixer, the profiles at $8.8 \%$ were characterized as a one-phase process, and the IPFS required a larger number of revolutions as filling level increased. To investigate the lubrication process in more detail, we analyzed the profile properties of the IPFS and the slope of the second phase (SSP) at each filling level and in each rotating mixer size. The SSP indicates the progression rate of the second phase, which gets higher with increasing the SSP as described in the previous report. ${ }^{62)}$

In the first phase, the relationship between the filling levels and the IPFS was investigated and is shown in Fig. 12. As the filling level increased, the IPFS increased, regardless of the rotating mixer size. The IPFS was linearly-correlated positively with the filing levels. It was speculated that as the amount of powder increased, the dispersion of the lubricant required more rotations. As the occupancy of the powder in the rotating mixer increased, the mixing efficiency decreased.

In the second phase, the relationship between the filling levels and the SSP was investigated and is shown in Fig. 13. The SSP reached a maximum value when the filling level was at the midpoint for the rotating mixer, regardless of the rotating mixer size. Unlike the first phase, the SSP was not correlated with the filling level. The optimal filling level where the SSP was at the maximum value was around $40 \%$. The progression rate of second phase at the $40 \%$ filling level was the highest.

In this study, we revealed the relationship between the property of each phase and the filling levels. We investigated the relationship between the property of each phase and the avalanche flow (avalanche time) and how this changes with the filling level. These results increase our understanding of the mechanism of the lubrication process.

Investigation in the Relationship between the Powder Flow and First Phase Process In the first phase, the IPFS was linearly-correlated positively with the filling level (Fig. 12). The relationship between the IPFS and the change of the avalanche flow changed by the filling level was investigated. The avalanche flow in the first phase was investigated based on avalanche flow time in the RPA.

The number of revolutions is an important parameter based on the thermal effusivity profile. We investigated how the number of avalanche flows per rotation changed at the various filling levels, using the result of avalanche time in the RPA. The avalanche number per rotation (ANR) was calculated by using the results of avalanche time in the RPA (Fig. 9). The ANR was calculated as the rotation speed of RPA divided by the avalanche time. The ANR changes as a function of the filling level is shown in Fig. 14. Because the amount of powder increased and the frequency of avalanche flow was lower, the ANR decreased as the filling levels increased. This investigation revealed that the ANR is linearly-correlated negatively with the filling levels.

The relationship between the IPFS and the ANR was investigated and is shown in Fig. 15. The IPFS value decreases with increasing the ANR. There is no clear impact of the rotating mixer size on the relationship between the ANR and the IPFS. 


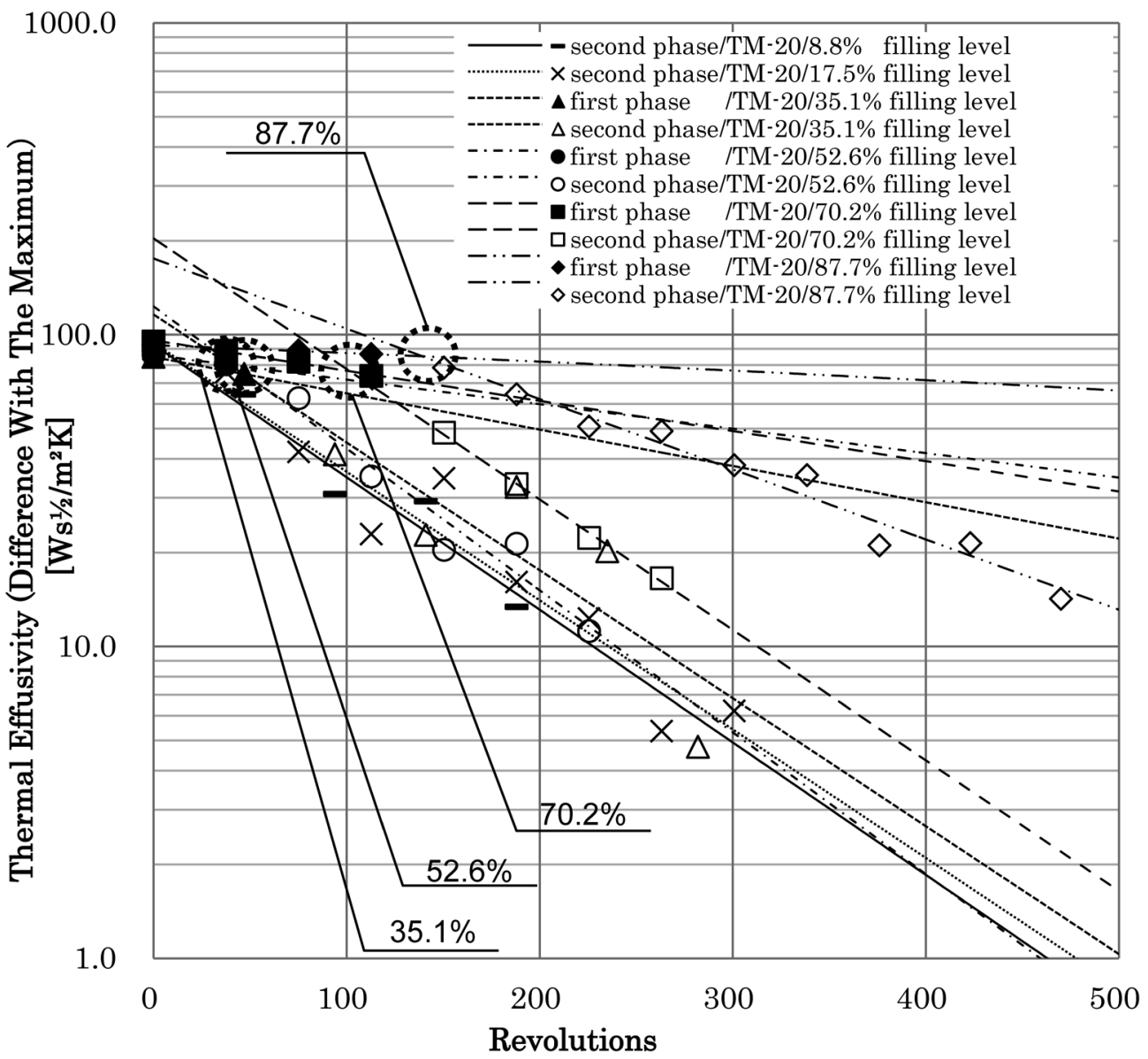

Fig. 11. Thermal Effusivity Data throughout the Lubrication Process Analyzed in Term of Two-Phase Process TM-20 mixer; the filling level $=8.8,17.5,35.1,52.6,70.2$ and $87.7 \%$.

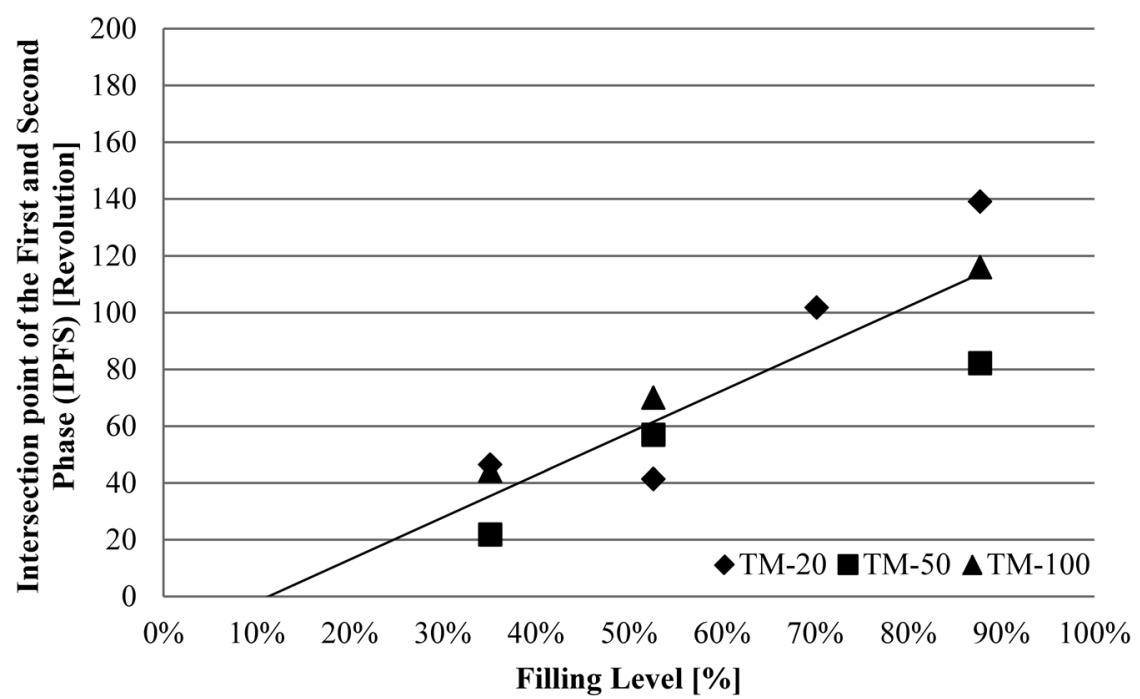

Fig. 12. Relationship of the Filling Level to the Intersection Point of the First and the Second Phases (IPFS) in TM-20, TM-50 and TM-100 Mixer

The data shows that the frequency in avalanche flow in the first phase is affected by the filling levels. The rotating mixer size did not impact this relationship, because the relative amount of powder in each mixer were similar.

Investigation in the Relationship between the Powder Flow and Second Phase Process In our previous studies, ${ }^{61,62)}$ it was proposed that the main purpose of the second phase is to coat the powder particles. The value of the thermal effusivity during the second phase was different than during the first phase. Powder movement in the rotating mixer was sufficient to coat the powder particles with lubricant in the second phase.

It was speculated that the mixing zone was more important than the stagnant zone for coating the powder particles with 


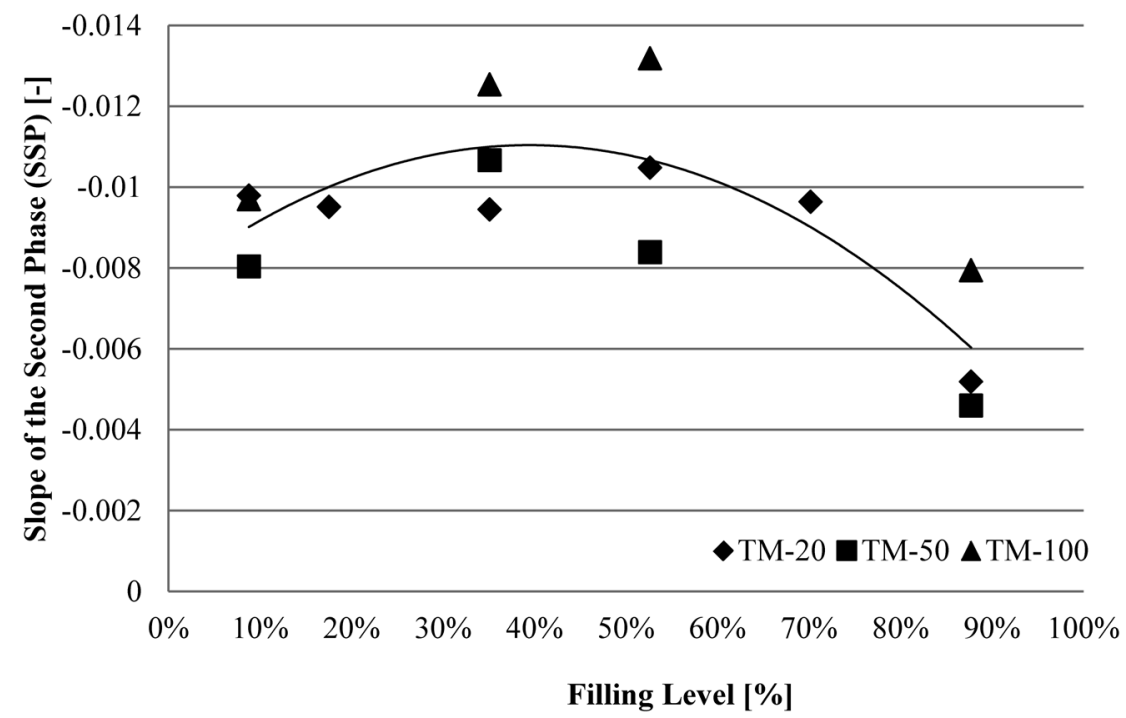

Fig. 13. Relationship of the Filling Level to the Slope of the Second Phase (SSP) in TM-20, TM-50 and TM-100 Mixer

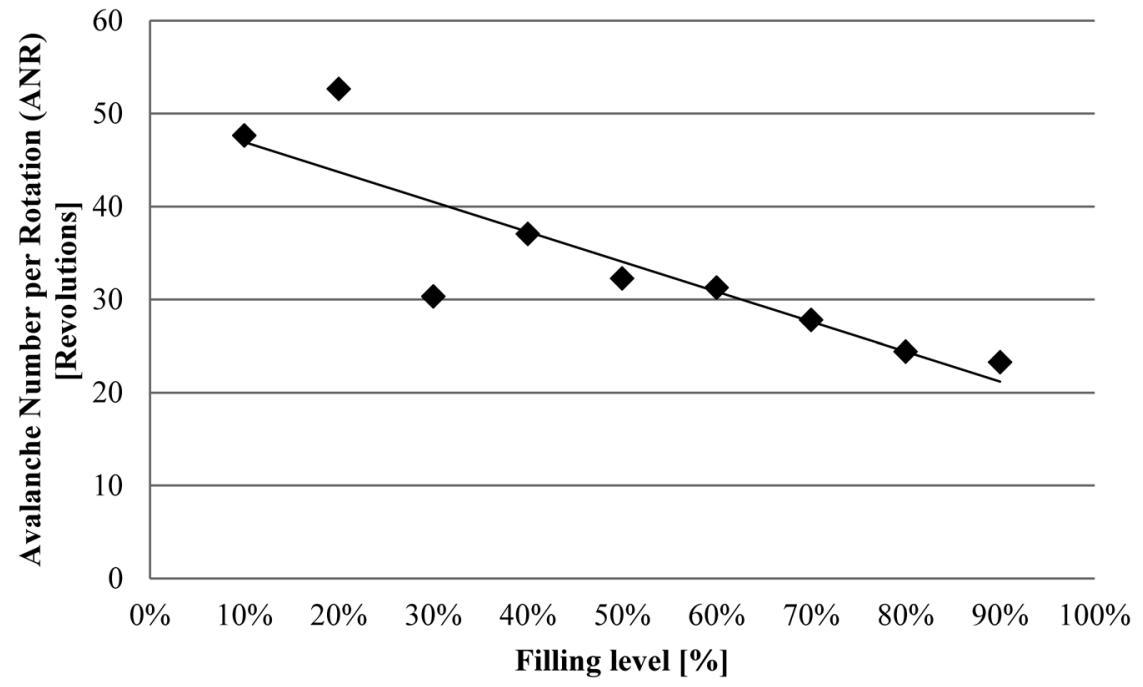

Fig. 14. Relationship of the Filling Level to Avalanche Number per Rotation (ANR) in the RPA

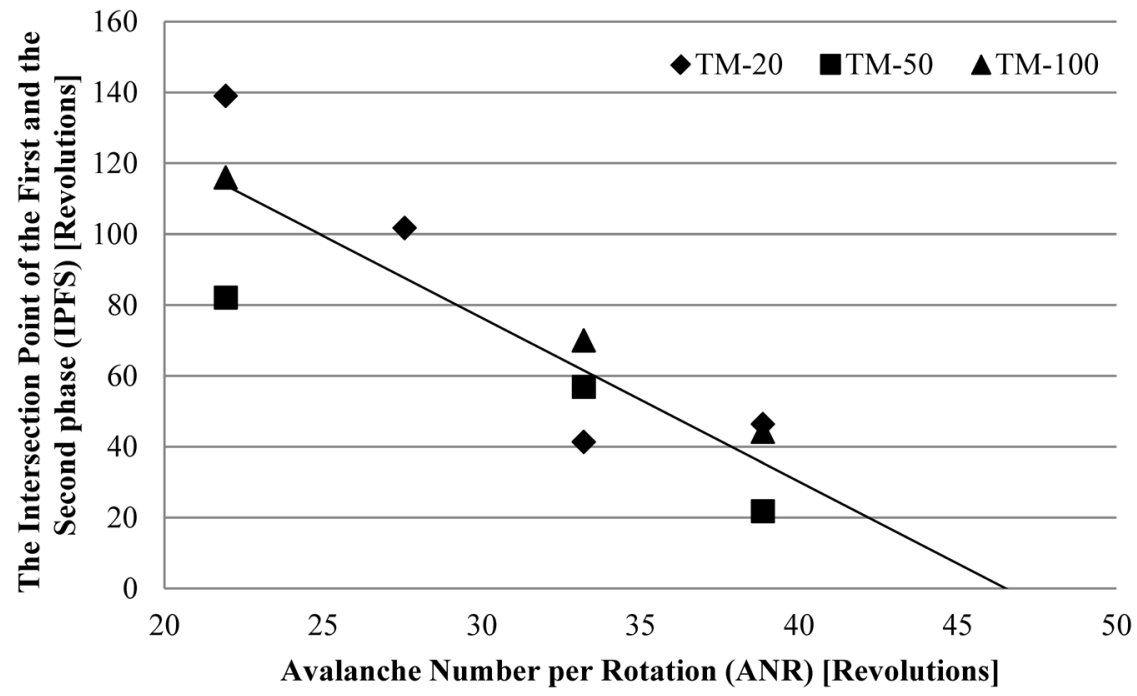

Fig. 15. Relationship of Avalanche Number per Rotation (ANR) to the Intersection Point of the First and the Second Phases (IPFS) in TM-20, TM-50 and TM-100 Mixer 


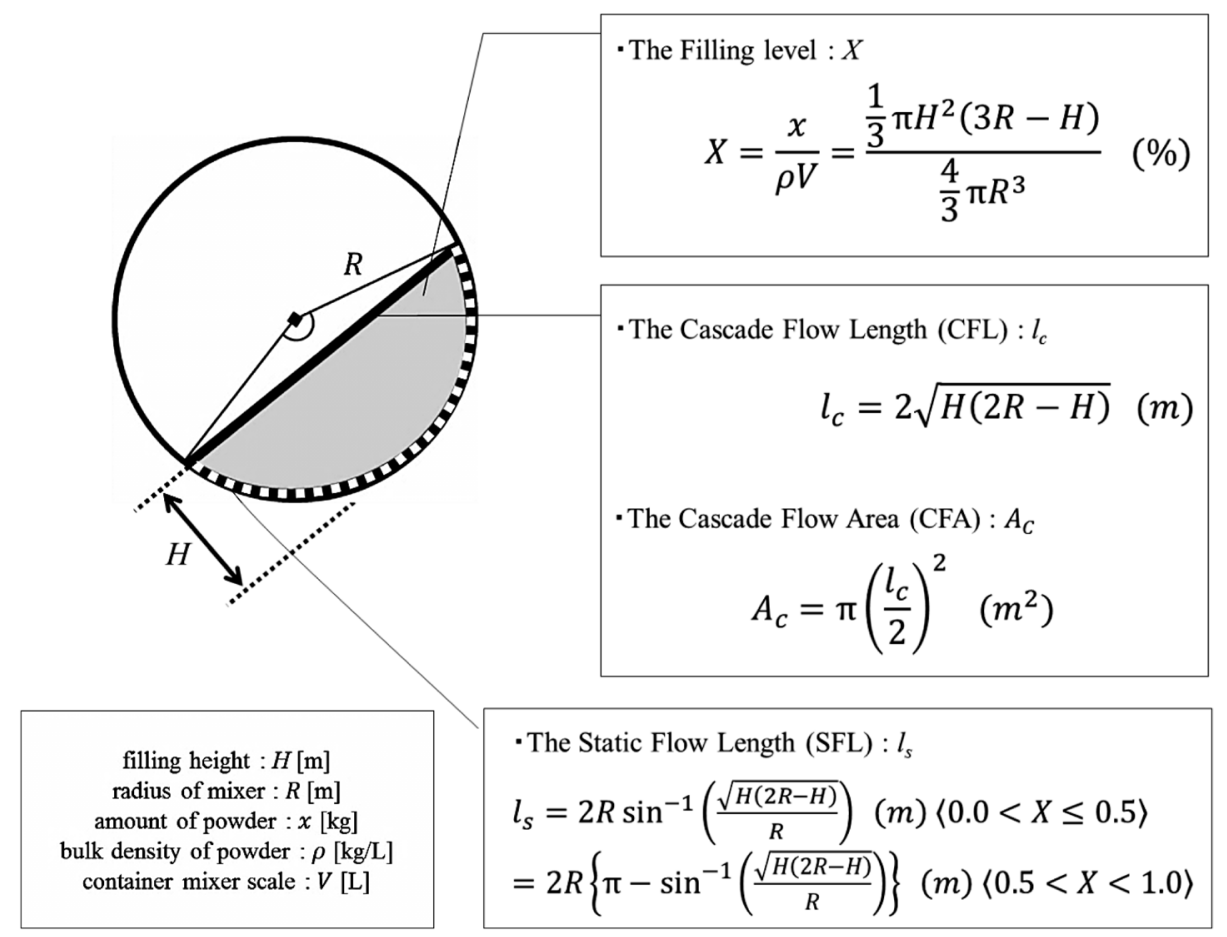

Fig. 16. Image of the Model of Lubrication Process

the dispersed lubricant. The relationships in both zones resulting from changes in the filling level and the rotating mixer size were investigated using a simple model. In this model, the rotating mixer is considered to have the shape of a sphere. The powder in the mixer during the lubrication process is represented as a cross section of a circle, and the volume of powders is represented as a part of a sphere. Similar cross sections can be achieved by dividing the sphere into multiple cross sectional portions. The mixing zone is represented as the chord of the circle. The cross section area at the center of the sphere was selected to be representative of the powder flow, to simplify the modeling and understanding. A side view of the representative cross-section is shown in Fig. 16. The length of the mixing zone is the length of chord, and is defined as the cascade flow length (CFL). The area of the mixing zone is the area of chord, and is defined as the cascade flow area (CFA). The length of stagnant zone is the length of circumference between the points where the chord contacts the wall, and is defined as the static flow length (SFL), which is shown as the dotted line in Fig. 16. The CFL, the CFA and the SFL are determined by the filling level. The filling level $(X)$ is calculated from Eq. 3. The CFL $\left(l_{c}\right)$, the CFA $\left(A_{c}\right)$ and the SFL $\left(l_{s}\right)$ can be calculated from Eqs. 4, 5 and 6;

The filling level;

$$
X=\frac{x}{\rho V}=\frac{(1 / 3) \pi H^{2}(3 R-H)}{(4 / 3) \pi R^{3}}
$$

The cascade flow length (CFL);

$$
l_{c}=2 \sqrt{H(2 R-H)} \quad(\mathrm{m})
$$

The cascade flow area (CFA);

$$
A_{c}=\pi\left(\frac{l_{c}}{2}\right)^{2} \quad\left(\mathrm{~m}^{2}\right)
$$

The static flow length (SFL);

$$
\begin{aligned}
l_{s} & =2 R \sin ^{-1}\left(\frac{\sqrt{H(2 R-H)}}{R}\right) \quad(\mathrm{m}) \quad(0.0<X \leq 0.5) \\
& =2 R\left\{\pi-\sin ^{-1}\left(\frac{\sqrt{H(2 R-H)}}{R}\right)\right\} \quad \text { (m) } \quad(0.5<X<1.0)
\end{aligned}
$$

where $x, \rho, V, H$, and $R$ are amount of powder $(\mathrm{kg})$, bulk density $(\mathrm{kg} / \mathrm{L})$, the rotating mixer size $(\mathrm{L})$, filling height $(\mathrm{m})$ and radius of the rotating mixer $(\mathrm{m})$, respectively. The CFL at the $50 \%$ filling level represents the maximum length which is equal to the diameter of the rotating mixer. The SFL at $100 \%$ filling level is equal to circumference of the rotating mixer. The CFA as a function of the filling level in the RPA can be calculated and is shown in Fig. 17. The value of CFA in the RPA reaches a maximum at the $50 \%$ filling level. The cascade flow area per rotation (CFAR) in the RPA at each filling level can be calculated by multiplication of the ANR value at each level (Fig. 14) and the CFA value at each level (Fig. 17), and is shown in Fig. 18. At filling levels less than 50\%, the CFA decreases but the frequency of avalanche flow increases. At filling levels more than $50 \%$, both the CFA and the frequency of avalanche flow decrease.

Since the shape of the RPA was similar to the TM-20, TM-50 and TM-100 mixer, the relationship between the filling level and the avalanche flow in the RPA was applied to the other rotating mixers. The CFAR for each rotating mixer was calculated based on the radius of each mixer. From the results of the SSP expressed in Fig. 13 and the calculated CFAR in each rotating mixer, the SSP was plotted as a function of each CFAR and is the shown in Fig. 19. Regardless of the rotating mixer size and the filling level, the SSP was linearly- 


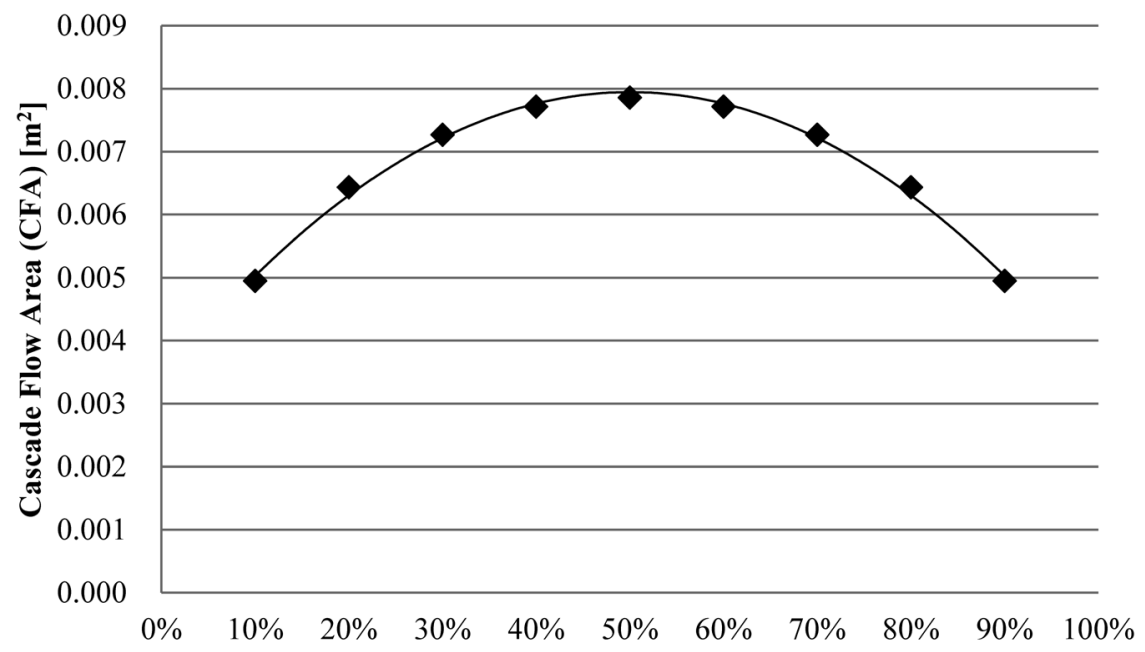

Filling Level [\%]

Fig. 17. Relationship of Filling Level to the Cascade Flow Area (CFA) in the RPA

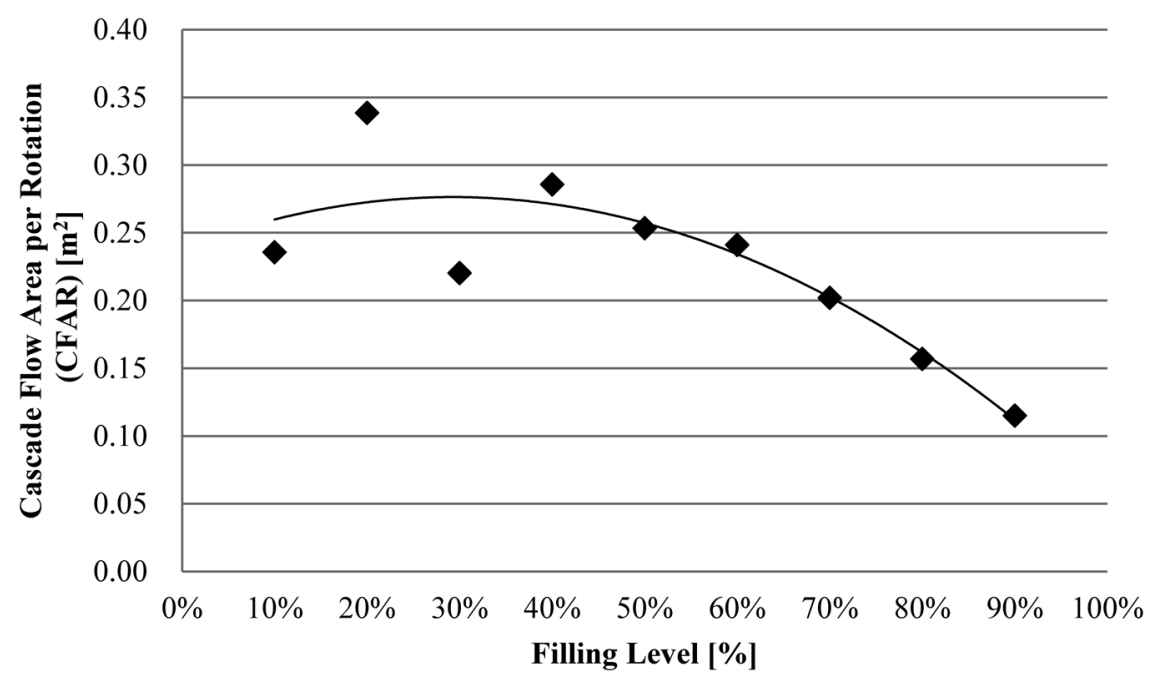

Fig. 18. Relationship of Filling Level to the Cascade Flow Area per Rotation (CFAR) in the RPA

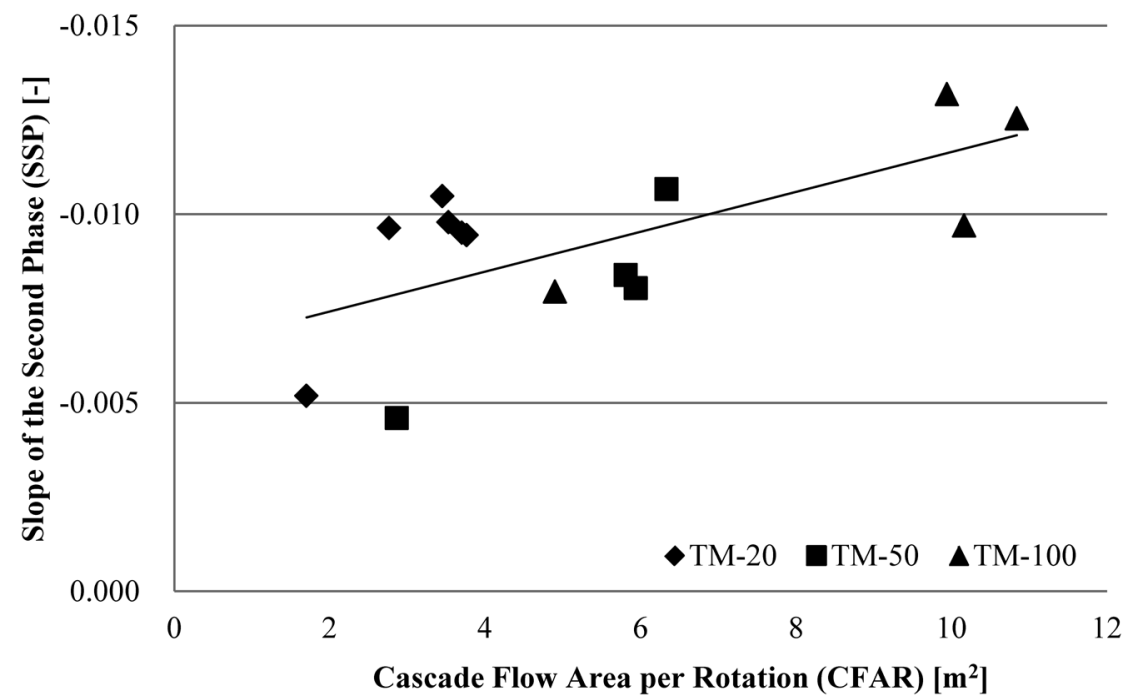

Fig. 19. Relationship of the Cascade Flow Area per Rotation (CFAR) to the Slope and the Second Phase (SSP) in TM-20, TM-50 and TM-100 Mixer 

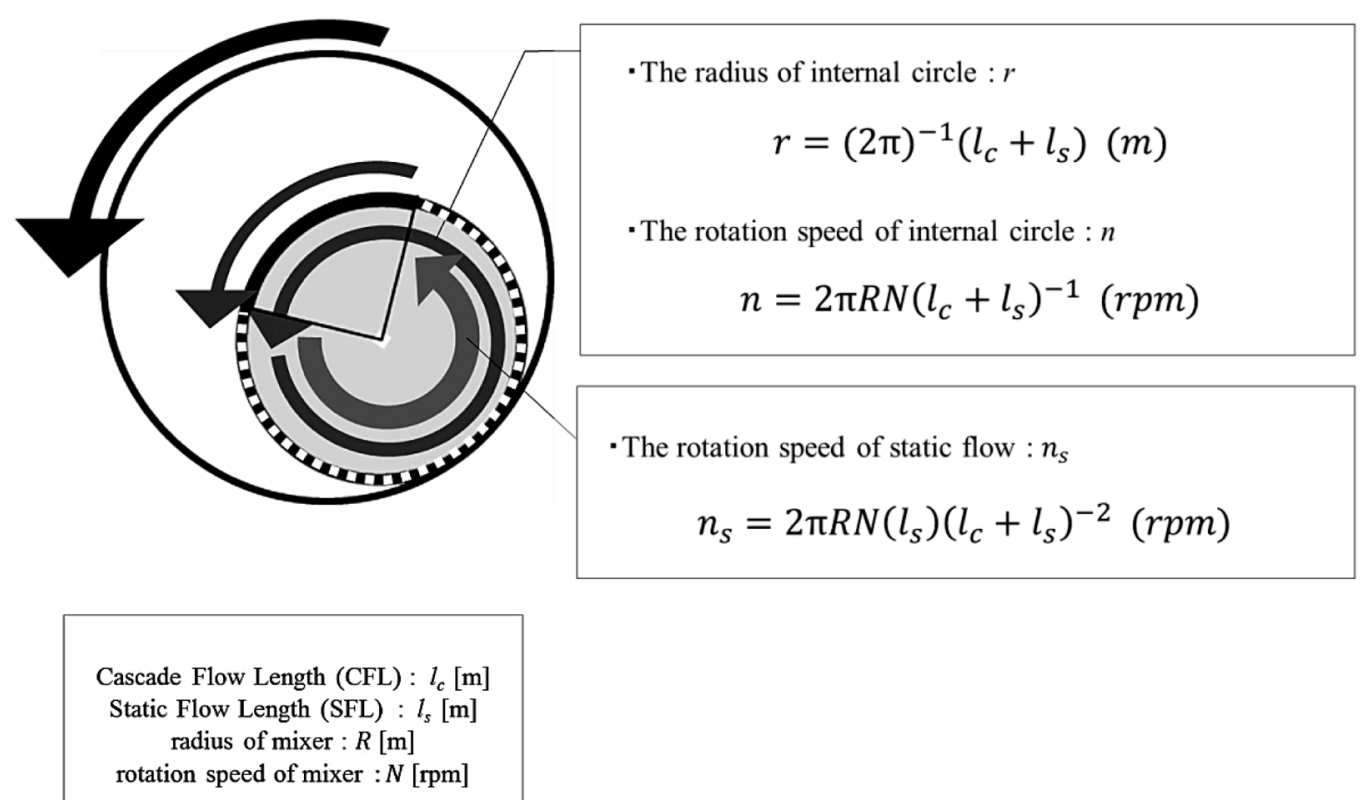

Fig. 20. Image of the Model of Lubrication Process-3

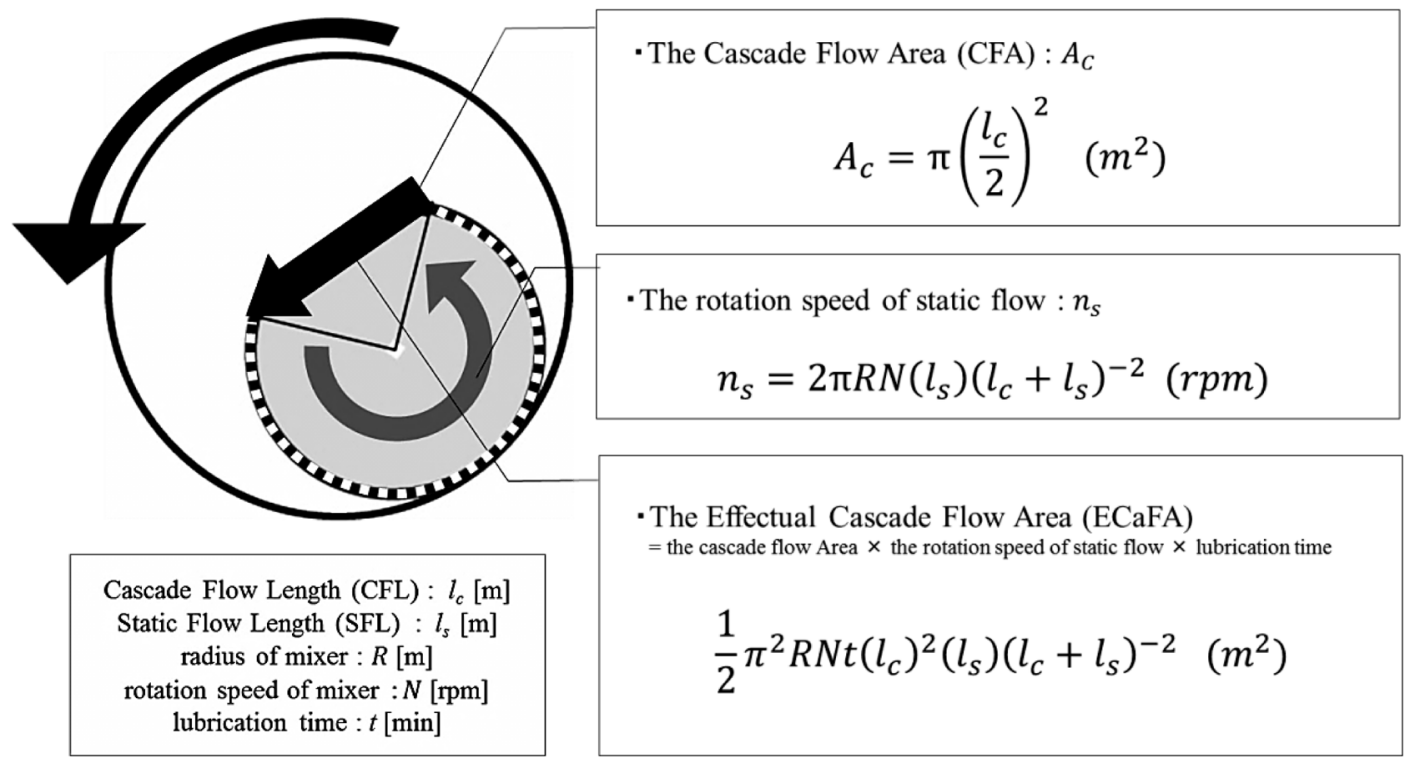

Fig. 21. Image of the Model of Lubrication Process-4

correlated negatively with the CFAR, and the SSP increases with increasing the CFAR. The CFAR is an important factor to consider in the lubrication process during the second phase, as the SSP was shown to influence the progression rate during the second phase.

The Model Establishment to Assess the Lubrication Process Obtained from the Profile of Thermal Effusivity The avalanche flow was shown to be an important factor to evaluate the lubrication process. However, the avalanche flow (the cascade flow and static flow) are influenced by the filling level, and the CFL, the CFA and the SFL are influenced by the filling level and the rotating mixer size. The lubrication rate during the second phase is related to the CFAR, regardless of the rotating mixer size and the filling level.

Using this knowledge, a model was established for the lubrication process. The model assumes that lubrication occurs in the mixing zone (the CFL and the CFA) and not in the stagnant zone (the SFL). We focused this balance of both zones, and tried to establish the model of the lubrication process to propose a simplified model that is shown in Figs. 20 and 21. The large circle represents the rotating mixer in this model, and the smaller internal circle represents the powder phase in the rotating mixer. The circumference (length of the outer boundary) for the internal circle was determined as the sum of the CFL and SFL. This internal circle is assumed to contact the large circle at a point, and the internal circle is assumed to rotate within the larger circle without slipping. As the filling level increases, the sum of the CFL and the SFL gets longer, as the internal circle becomes bigger, and the rotational speed of internal circle decreases.

Therefore the radius of internal circle " $r$ " can be calculated by the following Eq. 7; 


$$
r=(2 \pi)^{-1}\left(l_{c}+l_{s}\right) \quad(\mathrm{m})
$$

From this radius of internal circle and the rotation speed of mixer (rpm), the rotation speed " $n$ " (rpm) can be calculated as shown in Eq. 8;

$$
n=2 \pi R N\left(l_{c}+l_{s}\right)^{-1} \quad(\mathrm{rpm})
$$

The CFAR is an important factor to consider in the evaluation of the lubrication process as mention above. We focused on the CFA and the frequency of cascade flow. The frequency of cascade flow was determined by the balance between the CFL and SFL. During cascade flow, powder in the rotating mixer is lifted up by the distance of the SFL. Considering the frequency of cascade flow, the speed of portion of the powder in the SFL which contacted with the rotating mixer was more important than the portion in the CFL. Therefore, the rotation speed of static flow " $n_{s}$ " (rpm) are adjusted using Eq. 9;

$$
n_{s}=n \cdot \frac{l_{s}}{l_{c}+l_{s}}=2 \pi R N\left(l_{s}\right)\left(l_{c}+l_{s}\right)^{-2} \quad(\mathrm{rpm})
$$

From the multiplication of the cascade flow area, the rotation speed of the static flow and lubrication time " $t$ " (min), we establish the model of the effectual cascade flow aria (ECaFA) for the lubrication process. This model considers the total moving area in the cascade flow area. The ECaFA is calculated using Eq. 10;

The EcaFA;

$$
A_{c} \cdot n_{s} \cdot t=\frac{1}{2} \pi^{2} R N t\left(l_{c}\right)^{2}\left(l_{s}\right)\left(l_{c}+l_{s}\right)^{-2} \quad\left(\mathrm{~m}^{2}\right)
$$

By using the ECaFA, the profile of the lubrication process can be estimated regardless of the rotating mixer size, the filling level and the rotation speed. The thermal effusivity profile in the TM-50 mixer at a filling level of $35.1 \%$ was predicted from the data of the thermal effusivity profile in the TM-20 mixer at a filling level of $87.7 \%$. Both ECaFA was coincided and calculated as Eq. 11;

$$
\begin{aligned}
& \frac{1}{2} \pi^{2} R_{1} N_{1} t_{1}\left(l_{c_{1}}\right)^{2}\left(l_{s_{1}}\right)\left(l_{c_{1}}+l_{s_{1}}\right)^{-2} \\
& =\frac{1}{2} \pi^{2} R_{2} N_{2} t_{2}\left(l_{c_{2}}\right)^{2}\left(l_{s_{2}}\right)\left(l_{c_{2}}+l_{s_{2}}\right)^{-2}
\end{aligned}
$$

where $R_{1}, N_{1}, t_{1}, l_{c_{1}}$ and $l_{s_{1}}$ were the value about the TM-20 mixer at a filling level of $87.7 \%$ and $R_{2}, N_{2}, t_{2}, l_{c_{2}}$ and $l_{s_{2}}$ were the value about the TM-50 mixer at a filling level of $35.1 \%$. The calculation results using ECaFA method was shown in Table 1. Figure 22 shows the actual profile of thermal effusivity in the TM-20 mixer at the $87.7 \%$ filling level (A), the PREDICTED profile of thermal effusivity in the TM-50 mixer at the $35.1 \%$ filling level $(\square)$, and the ACTUAL profile of thermal effusiivity in the TM-50 mixer at the $35.1 \%$ filling level $(\square)$. The profiles of both actual and predicted profile of TM-50 mixer were almost the same. Other examples using similar method, the thermal effusivity profile in the TM-20 mixer at a filling level of $70.2 \%$ was predicted from the data of the thermal effusivity profile in the TM-100 mixer at a filling level of $8.8 \%$. Figure 23 shows the actual profile of thermal effusivity in the TM-100 mixer at the $8.8 \%$ filling level $(\diamond)$, the PREDICTED profile of thermal effusivity in the TM-20 mixer at the $70.2 \%$ filling level $(\bigcirc)$, and the ACTUAL profile of thermal effusiivity in the TM-20 mixer at the $70.2 \%$ filling level (O). We ran simulations at other conditions and confirmed this estimation was valid for other scenarios as

\begin{tabular}{|c|c|c|c|c|c|c|c|c|}
\hline \multicolumn{4}{|c|}{$\begin{array}{c}\text { TM-20 } \\
\text { Fillng level }(\%)=87.7 \\
R_{1}(\mathrm{~m})=0.185 \\
N_{1}(\mathrm{rpm})=18.8\end{array}$} & \multicolumn{5}{|c|}{$\begin{array}{c}\text { TM-50 } \\
\text { Fillng level }(\%)=35.1 \\
R_{2}(\mathrm{~m})=0.240 \\
N_{2}(\mathrm{rpm})=16.5\end{array}$} \\
\hline \multicolumn{4}{|c|}{$\frac{1}{2} \pi^{2} R_{2}\left(l_{c_{1}}\right)^{2}\left(l_{s_{1}}\right)\left(l_{c_{1}}+l_{s_{1}}\right)^{-2}=0.0558$} & \multicolumn{5}{|c|}{$\frac{1}{2} \pi^{2} R_{2}\left(l_{c_{2}}\right)^{2}\left(l_{s_{2}}\right)\left(l_{c_{2}}+l_{s_{2}}\right)^{-2}=0.1354$} \\
\hline \multicolumn{4}{|c|}{ Actual data } & \multicolumn{2}{|c|}{ Prediction data } & \multicolumn{3}{|c|}{ Actual data } \\
\hline$t_{1}(\min )$ & Revolutions & $\mathrm{E}\left(\mathrm{Ws}^{1 / 2} / \mathrm{m}^{2} \mathrm{~K}\right)$ & $\mathrm{ECaFA}$ & Revolutions & $\mathrm{E}\left(\mathrm{Ws}^{1 / 2} / \mathrm{m}^{2} \mathrm{~K}\right)$ & $t_{2}(\min )$ & Revolutions & $\mathrm{E}\left(\mathrm{Ws}^{1 / 2} / \mathrm{m}^{2} \mathrm{~K}\right)$ \\
\hline 0 & 0.0 & 259.18 & 0.0 & 0.0 & 259.18 & 0 & 0.0 & 257.39 \\
\hline 2 & 37.6 & 261.95 & 2.1 & 15.5 & 261.95 & 2 & 33.0 & 267.32 \\
\hline 4 & 75.2 & 263.03 & 4.2 & 31.0 & 263.03 & 4 & 66.0 & 284.19 \\
\hline 6 & 112.8 & 265.41 & 6.3 & 46.5 & 265.41 & 6 & 99.0 & 310.81 \\
\hline 8 & 150.4 & 273.60 & 8.4 & 62.0 & 273.60 & 8 & 132.0 & 321.84 \\
\hline 10 & 188.0 & 287.54 & 10.5 & 77.5 & 287.54 & 10 & 165.0 & 326.88 \\
\hline 12 & 225.6 & 301.22 & 12.6 & 93.0 & 301.22 & 12 & 198.0 & 327.97 \\
\hline 14 & 263.2 & 302.87 & 14.7 & 108.6 & 302.87 & 14 & 231.0 & 341.36 \\
\hline 16 & 300.8 & 313.82 & 16.8 & 124.1 & 313.82 & 16 & 264.0 & 342.25 \\
\hline 18 & 338.4 & 316.54 & 18.9 & 139.6 & 316.54 & 18 & 297.0 & 339.49 \\
\hline 20 & 376.0 & 330.86 & 21.0 & 155.1 & 330.86 & 20 & 330.0 & 338.33 \\
\hline 22.5 & 423.0 & 330.51 & 23.6 & 174.5 & 330.51 & 22.5 & 371.3 & 338.74 \\
\hline 25 & 470.0 & 337.73 & 26.2 & 193.9 & 337.73 & 25 & 412.5 & 341.09 \\
\hline 30 & 564.0 & 333.30 & 31.5 & 232.6 & 333.30 & 30 & 495.0 & 340.45 \\
\hline 40 & 752.0 & 337.07 & 42.0 & 310.2 & 337.07 & 40 & 660.0 & 334.00 \\
\hline 50 & 940.0 & 346.91 & 52.5 & 387.7 & 346.91 & 50 & 825.0 & 334.78 \\
\hline
\end{tabular}
well. When the value of thermal effusivity is similar, the physical properties of the powder and the tablet were also very similar. ${ }^{61)}$ Therefore physical properties of the blended powder

Table 1. Thermal Effusivity Data throughout the Lubrication Process Using the Method of the Effectual Cascade Flow Area (ECaFA) - Example 1 (Scale-Up)- 


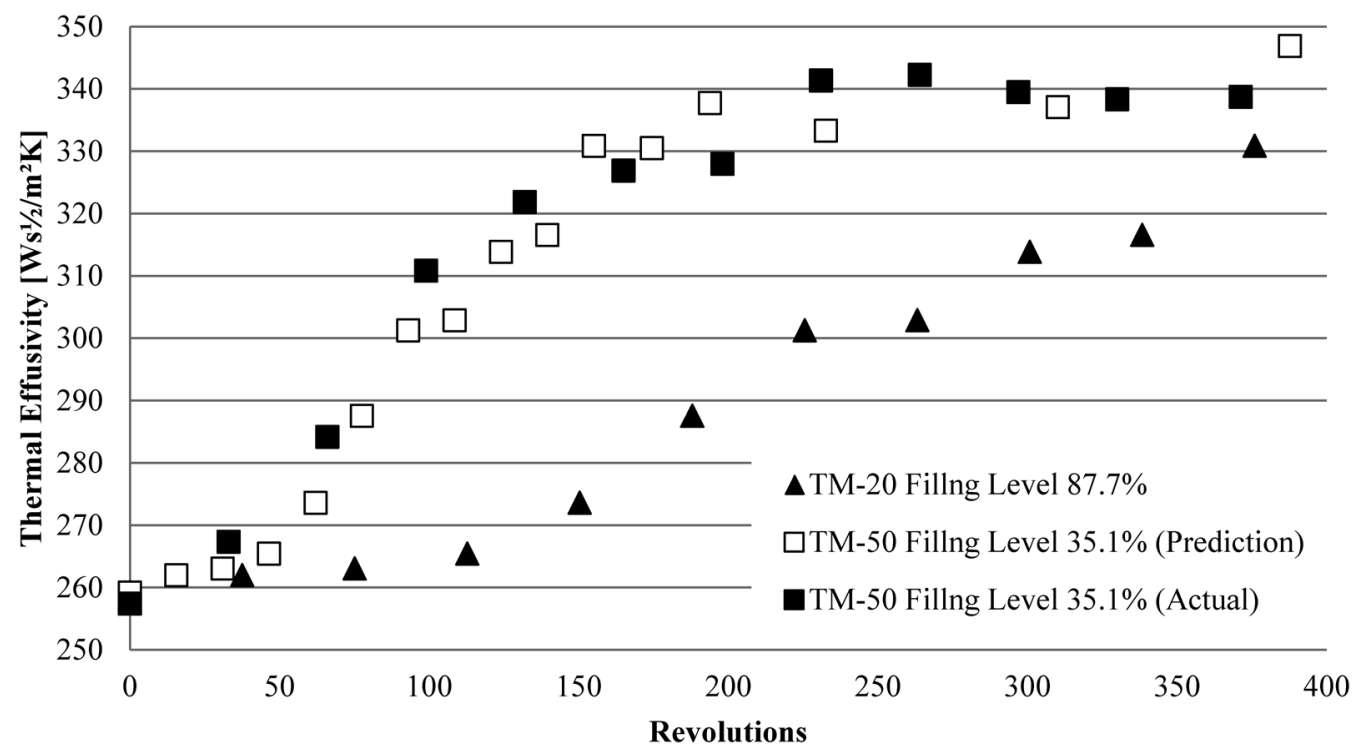

Fig. 22. Thermal Effusivity Data throughout the Lubrication Process Using the Method of the Effectual Cascade Flow Area (ECaFA)—Example 1 (Scale-Up)-

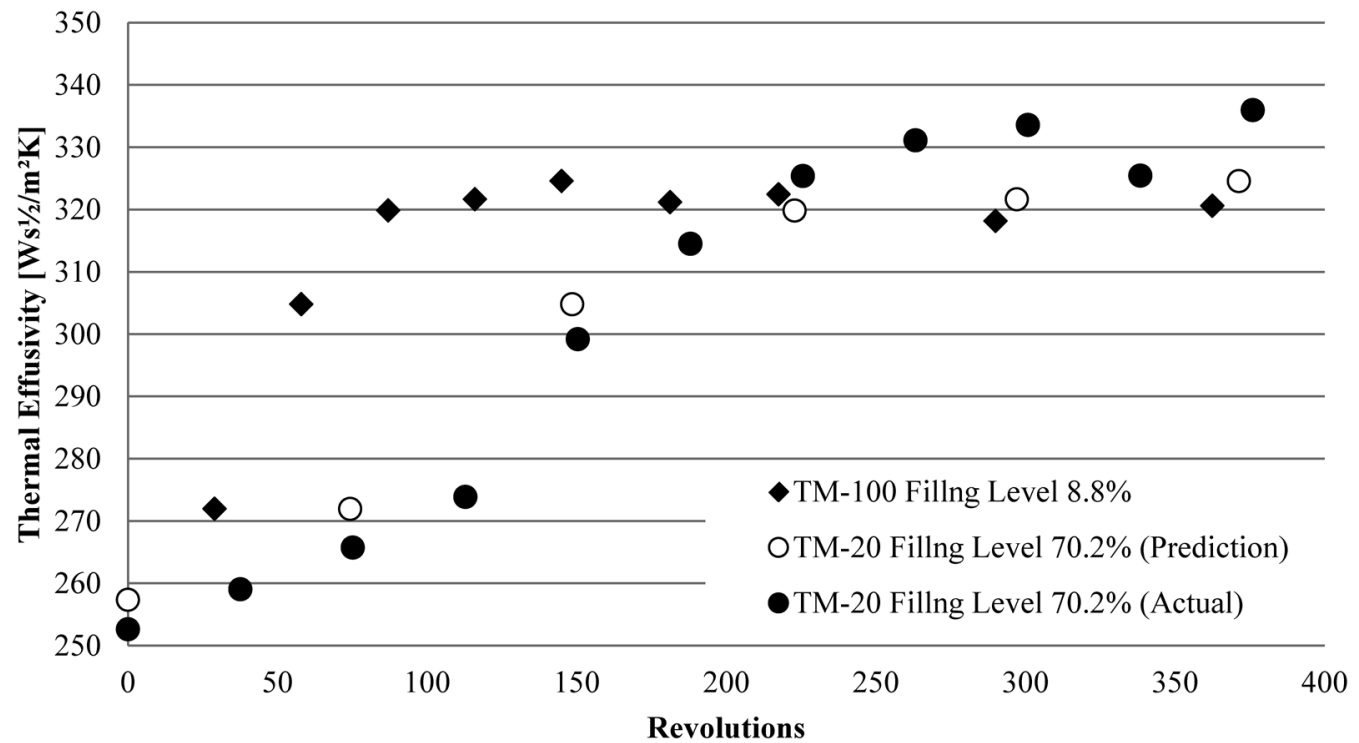

Fig. 23. Thermal Effusivity Data throughout the Lubrication Process Using the Method of the Effectual Cascade Flow Area (ECaFA) - Example 2 (Scale-Down)-

and tablet can also be estimated. This study has shown that the ECaFA method is very useful. Future efforts could also consider applicability of the ECaFA strategy to other rotating mixer types and sizes.

\section{Conclusion}

This study focused on the lubrication process which is the blending of a minor component, using the thermal effusivity sensor which is able to capture the lubrication process in detail. The thermal effusivity was used to investigate how the lubrication process is affected by changes in the blending conditions including the filling level and the rotating mixer size. In addition, an investigation of the movement of the powder flow using the RPA was conducted in an attempt to understand the mechanism of the lubrication process. During this study, the first and the second phases were observed in the lubrication process. The lubrication process was affected by the filling levels but not by the rotating mixer size. In the evaluation of powder flow in the rotating mixer, the frequency of avalanche flow in the rotating mixer decreased as the filling level increased. For an adequate lubrication process, the avalanche flow which involves slipping on superficial layer is important. The proportion of the mixing zone and the stagnant zone are determined by the filling levels and this has a significant impact on mixing efficiency. In the first phase, there was an inverse correlation between the IPFS value and the ANR and there is no clear impact of the rotating mixer size on the relationship between the ANR and the IPFS. In the second phase, the SSP was linearly-correlated negatively with the CFAR regardless of the rotating mixer size and the filling level. The CFAR is an important factor to consider in the lubrication process during the second phase, as the SSP was shown to influence the progression rate during the second phase. 
By considering the detailed phenomenon of lubrication process in the first and second phase, we established the ECaFA method. By using this method, the profile of lubrication process can be estimated regardless of the rotating mixer size, the filling level or the rotation speed. It is then assumed that lubrication profile at the commercial scale will be predicted from that at laboratory scale, and the change of filling level can be also estimated from the current data.

Acknowledgment We are grateful to Higuchi Inc. for its offer of Thermal Effusivity Sensor and Nihon Rufuto Co., Ltd. for its offer of REVOLUTION ${ }^{\circledR}$ Powder Analyzer.

Conflict of Interest The authors declare no conflict of interest.

\section{References}

1) Armstrong N. A., Encyclopedia of Pharmaceutical Technology, 2002, 2713-2732 (2002).

2) Tardos G. I., Khan M. I., Mort P. R., Powder Technol., 94, 245-258 (1997).

3) Lindberg N.-O., Leander L., Reenstierna B., Drug Dev. Ind. Pharm., 8, 775-782 (1982).

4) Holm P., Schaefer T., Kristensen H. G., Powder Technol., 43, 213223 (1985).

5) Sirois P. J., Craig G. D., Pharm. Dev. Technol., 5, 365-374 (2000).

6) Iskandarani B., Shiromani P. K., Clair J. H., Drug Dev. Ind. Pharm., 27, 651-657 (2001)

7) Radeke C. A., Glasser B. J., Khinast J. G., Chem. Eng. Sci., 65, 6435-6442 (2010).

8) Faure A., York P., Rowe R. C., Eur. J. Pharm. Biopharm., 52, 269-277 (2001)

9) Shah A. C., Mlodozeniec A. R., J. Pharm. Sci., 66, 1377-1382 (1977).

10) Roblot-Treupel L., Puisieux F., Int. J. Pharm., 31, 131-136 (1986).

11) Hafeez Hussain M. S., York P., Timmins P., Int. J. Pharm., 42, 89-95 (1988)

12) Wang J., Wen H., Desai D., Eur. J. Pharm. Biopharm., 75, 1-15 (2010).

13) Jarosz P. J., Parott E. L., Drug Dev. Ind. Pharm., 10, 259-273 (1984)

14) Kikuta J., Kitamori N., Drug Dev. Ind. Pharm., 20, 343-355 (1994).

15) Rao K. P., Chawla G., Kaushal A. M., Bansal A. K., Pharm. Dev. Technol., 10, 423-437 (2005).

16) Ertel K. D., Carstensen J. T., J. Pharm. Sci., 77, 625-629 (1988).

17) Fukui E., Miyamura N., Kobayashi M., Int. J. Pharm., 216, 137-146 (2001)

18) Billany M. R., Richards J. H., Drug Dev. Ind. Pharm., 8, 497-511 (1982).

19) Levy G., Gumtow R. H., Journal of Pharmaceutical Sciences, 52, 1139-1144 (1963).

20) Harnby N., Pharm. Sci. Technol. Today, 3, 303-309 (2000).

21) Sudah O. S., Coffin-Beach D., Muzzio F. J., Int. J. Pharm., 247, 57-68 (2002).

22) Yano T., Terashita K., Powder Technology Japan, 11, 392-399 (1974).

23) Lemieux M., Bertrand F., Chaouki J., Gosselin P., Chem. Eng. Sci., 62, 1783-1802 (2007).

24) Brone D., Muzzio F. J., Powder Technol., 110, 179-189 (2000).

25) Van Puyvelde D. R., Young B. R., Wilson M. A., Schmidt S. J., Powder Technol., 106, 183-191 (1999).

26) Santomaso A., Olivi M., Canu P., Chem. Eng. Sci., 59, 3269-3280 (2004).

27) Muzzio F. J., Alexander A. W., Pharmaceutical Technology, Scaling Up Manufacturing, s34-s44 (2005).
28) Bolhuis G. K., De Jong S. W., Lerk C. F., Dettmers H., Drug Dev. Ind. Pharm., 13, 1547-1567 (1987).

29) Bolhuis G. K., De Jong S. W., Van Kamp H. V., Dettmers H., Pharm. Tech. Jpn., 3, 877-883 (1987).

30) Kushner J. IV, Moore F., Int. J. Pharm., 399, 19-30 (2010).

31) Van der Watt J. G., De Villiers M. M., Eur. J. Pharm. Biopharm., 43, 91-94 (1997).

32) Maeda J., Suzuki T., Takayama K., Chem. Pharm. Bull., 60, $1155-$ 1163 (2012).

33) El Hagrasy A. S., Chang S., Desai D., Kiang S., Journal of Pharmaceutical Innovation, September/October, 37-42 (2006).

34) Portillo P. M., Ierapetritou M. G., Muzzio F. J., Powder Technol., 182, 368-378 (2008).

35) Vanarase A. U., Alcalá M., Jerez Rozo J. I., Muzzio F. J., Romañach R. J., Chem. Eng. Sci., 65, 5728-5733 (2010).

36) Vanarase A. U., Muzzio F. J., Powder Technol., 208, 26-36 (2011).

37) Sekulic S. S., Ward W. H. II, Brannegan D. R., Stanley E. D., Evans C. L., Sciavolino S. T., Hailey P. A., Aldridge P. K., Anal. Chem., 68, 509-513 (1996).

38) Hailey P. A., Doherty P., Tapsell P., Oliver T., Aldridge P. K., J. Pharm. Biomed. Anal., 14, 551-559 (1995).

39) Blanco M., Gozález Bañó R., Bertran E., Talanta, 56, 203-212 (2002).

40) Wargo D. J., Drennen J. K., J. Pharm. Biomed. Anal., 14, 1415-1423 (1996).

41) El-Hagrasy A. S., Morris H. R., D’amico F., Lodder R. A., Drennen J. K. III, J. Pharm. Sci., 90, 1298-1307 (2001).

42) Arratia P. E., Duong N.-H., Muzzio F. J., Godbole P., Lange A., Reynolds S., Powder Technol., 161, 202-208 (2006).

43) Abe H., Otsuka M., Drug Dev. Ind. Pharm., 38, 412-419 (2012).

44) Duong N.-H., Arratia P., Muzzio F., Lange A., Timmermans J., Reynolds S., Drug Dev. Ind. Pharm., 29, 679-687 (2003).

45) Terashita K., Pharm. Tech. Jpn., 28, 2407-2410 (2012).

46) Nakagawa H., Kano M., Hasebe S., Suzuki T., Wakiyama N., Int. J. Pharm., 441, 402-413 (2013).

47) Terashita K., Sato T., Pharm. Tech. Jpn., 24, 613-620 (2008).

$48)$ Donoso M., Kildsig D. O., Ghaly E. S., Pharm. Dev. Technol., 8, 357-366 (2003).

49) Otsuka M., Yamane I., J. Pharm. Sci., 95, 1425-1433 (2006).

50) Otsuka M., Yamane I., J. Pharm. Sci., 98, 4296-4305 (2009).

51) Andersson M., Josefson M., Langkilde F. W., Wahlund K.-G., J. Pharm. Biomed. Anal., 20, 27-37 (1999).

52) Andersson M., Folestad S., Gottfries J., Johansson M. O., Josefson M., Wahlund K.-G., Anal. Chem., 72, 2100-2018 (2000).

53) Kirsch J. D., Drennen J. K., Pharm. Res., 13, 234-237 (1996).

54) Yonemochi E., Netsu Sokutei, 35, 180-184 (2008).

55) Mathews L., Chandler C., Dipali S., Adusumilli P., Lech S., Daskalakis S., Mathis N., Pharmaceutical Technology, APRIL 2002, 26, 80-84 (2002).

56) Ghorab M. K., Chatlapalli R., Hasan S., Nagi A., AAPS PharmSciTech, 8, E155-E161 (2007).

57) Roy Y., Closs S., Mathis N., Nieves E., Pharmaceutical Technology, PROCESS ANALYTICAL TECHNOLOGY 2004, 28, 21-28 (2004).

58) Roy Y., Mathis N., Tablets and Capsules, 3, 38-47 (2005).

59) Terashita K., Pharm. Tech. Jpn., 28, 921-926 (2012).

60) Harris H., Sorensen D. N., Journal of Pyrotechnics, 25, 49-54 (2007).

61) Uchiyama J., Kato Y., Uemoto Y., Drug Dev. Ind. Pharm., 40, 999-1004 (2014)

62) Uchiyama J., Aoki S., Drug Dev. Ind. Pharm., in press.

63) Mullarney M. P., Hancock B. C., Carlson G. T., Ladipo D. D., Langdon B. A., Int. J. Pharm., 257, 227-236 (2003).

64) "Flowability testing with the revolution powder analyzer.": 〈http:// www.mercsci.com/flowability.htm), cited 6 June, 2014.

65) Inoue I., Yamaguchi K., Sato K., Chemical Engineering, 34, $1323-$ 1329 (1970). 